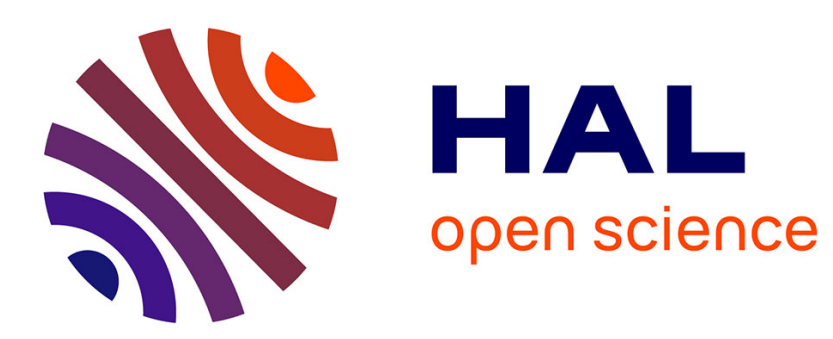

\title{
La régulation asymétrique : un mécanisme de financement des coûts échoués irrécupérables
}

Cédric Clastres

\section{To cite this version:}

Cédric Clastres. La régulation asymétrique : un mécanisme de financement des coûts échoués irrécupérables. Revue d'Economie Politique, 2016, 126 (1), pp.89-126. 10.3917/redp.261.0089 . hal01279582

HAL Id: hal-01279582

https://hal.univ-grenoble-alpes.fr/hal-01279582

Submitted on 26 Feb 2016

HAL is a multi-disciplinary open access archive for the deposit and dissemination of scientific research documents, whether they are published or not. The documents may come from teaching and research institutions in France or abroad, or from public or private research centers.
L'archive ouverte pluridisciplinaire HAL, est destinée au dépôt et à la diffusion de documents scientifiques de niveau recherche, publiés ou non, émanant des établissements d'enseignement et de recherche français ou étrangers, des laboratoires publics ou privés. 


\title{
La régulation asymétrique : un mécanisme de financement des coûts échoués irrécupérables
}

\author{
Cédric Clastres * *
}

5 juin 2015

\section{Résumé}

Les régulations asymétriques modifient les contextes réglementaires et concurrentiels des industries de réseaux. L'efficacité économique, la collusion ou le financement des coûts échoués irrécupérables sont autant de questions qui émergent avec ces mesures réglementaires. Les régulateurs se doivent d'adapter leur politique de régulation afin d'internaliser ces questions et de maximiser le welfare. Nous montrons à l'aide d'un modèle oligopolistique "à la Cournot" qu'il est possible pour un régulateur de financer les coûts échoués irrécupérables et de conserver l'efficacité productive. Le succès de cette politique dépend de l'efficacité de l'OH et du prix des sources alternatives d'approvisionnement.

Mots Clés : Régulation asymétrique, coûts échoués irrécupérables, concurrence forcée, contraintes de capacités.

Codes de classification JEL : L13, L51, L97.

Title : Funding sunk stranded costs with asymetric regulation

* Je remercie les deux rapporteurs anonymes ainsi que Laurent David, Stéphane Lemarié et Haikel Khalfallah pour leurs suggestions et commentaires pertinents. Je reste seul responsable des éventuelles erreurs ou maladresses.

${ }^{\dagger}$ Univ. Grenoble Alpes, PACTE, EDDEN, F-38000 Grenoble, France

${ }^{\ddagger}$ CNRS, PACTE, EDDEN, F-38000 Grenoble, France Email : cedric.clastres@upmfgrenoble.fr 


\begin{abstract}
Asymetric regulation in networks industries have impacted regulatory and competitive contexts. Questions of economic efficiency, collusion or fundings of sunk stranded costs go with asymetric regulation. Thus, regulators must adapt their policies to internalise these questions and to maximise the welfare. We show with a Cournot model regulators could fund sunk stranded costs, keeping allocative efficiency constant. Positive results on welfare rely on incumbent's efficiency and on prices of alternative supplies.
\end{abstract}

Keywords : Asymetric regulation, sunk stranded costs, forced competition, capacity constraints.

JEL Classification : L13, L51, L97. 


\section{Introduction}

L'ouverture à la concurrence des industries de réseaux se réalise en séparant voire déintégrant les différents maillons industriels. Au côté du secteur régulé du transport (Percebois [2001]), l'accès aux inputs (électricité, gaz naturel) revendus sur le marché final concurrentiel devient source d'attention. Les raisons de cet intérêt peuvent être nombreuses, comme par exemple le manque de liquidité des marchés de gros, la saturation des infrastructures de transport, le délai de mise en place de nouvelles infrastructures de production ou d'importation, la concentration de l'amont ou encore l'efficacité des opérateurs en place.

Pour faciliter cet accès, certains régulateurs ont décidé de mettre en place des Régulations Asymétriques (RA) pour faciliter la concurrence sur le marché de la fourniture. Schankerman [1996] définit une régulation symétrique comme une régulation qui donne à tous les participants d'un marché les mêmes règles pour se concurrencer et réaliser leur activité. Chacun d'eux doit recevoir les mêmes signaux de prix et tous se doivent de respecter les mêmes obligations et contraintes. Une RA se définit comme un ensemble de règles édictées par le régulateur et qui ne s'appliquent qu'à un ou plusieurs opérateurs. L'objectif de ces mesures est de promouvoir la concurrence sur le marché final en augmentant le nombre d'acteurs actifs dans le secteur.

La Commission européenne juge positivement ces mesures car elles permettent de réduire les positions dominantes observées (European Commission [2007]). Cependant, aucune de ses décisions formelles ou textes réglementaires n'oblige à les appliquer. Ces RA ont été généralement mises en place nationalement, par les régulateurs des diverses industries de réseaux. Elles sont temporaires et ne servent qu'à aider provisoirement les concurrents à pénétrer le marché le temps de développer leurs sources d'approvisionnement. Cette concurrence forcée permet au concurrent d'être actif sur le marché. Cependant, cette nouvelle situation concurrentielle entraîne l'apparition de coûts échoués irrécupérables pour l'Opérateur Historique $(\mathrm{OH})$. Ces coûts échoués irrécupérables doivent être financés et il est parfois optimal pour le consom- 
mateur que ce financement soit effectué par le biais de la régulation (Baumol et Siddak [1995]), financement que la théorie sur la RA ne mentionne pas.

Nous verrons dans une deuxième section que les avis sur l'efficacité des RA sont partagés. En effet, les impacts sur les incitations à la collusion, sur l'efficacité des concurrents et le financement des coûts échoués irrécupérables posent question. Nous montrerons dans une troisième section que le régulateur peut réduire l'impact de ces coûts échoués irrécupérables en adaptant sa politique de régulation pour effectuer des transferts entre acteurs. Des équilibres théoriques maximisant le welfare seront ainsi calculés. Dans une quatrième section, nous introduirons une source alternative d'approvisionnement. Cette dernière modifie cette politique de régulation car le concurrent peut réaliser des arbitrages entre les deux sources d'approvisionnement selon le niveau des prix relatifs.

\section{La régulation asymétrique et la concurrence forcée}

\subsection{Les expériences dans les industries de réseaux}

Dans le secteur américain des télécommunications, les $\mathrm{OH}$ devaient tarifer les services locaux et les appels de longues distances à un prix régulé et publié. Les concurrents pouvaient alors pratiquer des stratégie d'écrémage (Schankerman et Waverman [1997], Perrucci et Cimatoribus [1997]). Sur les marchés gaziers, des régulateurs (Grande-Bretagne, Italie, Espagne, France, Autriche, etc.) ont adopté des mesures de gas release. Les $\mathrm{OH}$ devaient rétrocéder une partie de leurs approvisionnements contractés à long terme (contrats Take or Pay) à leurs concurrents. Le prix de rétrocession pouvait être un prix moyen ou déterminé par un système d'enchères (Clastres [2005], Clastres et David [2009]). L'information sur la détermination de la quantité globale rétrocédée était moins transparente, la méthode n'étant pas publiée. La seule information était que cette quantité rétrocédée permettait de rendre 
les concurrents actifs sur le marché. La répartition de cette quantité entre chaque candidat était connue et effectuée soit au travers d'une enchère (avec une quantité plafond pour l'achat d'un acteur), soit au prorata des quantités demandées par les candidats. Sur les marchés électriques, les $\mathrm{OH}$ devaient rétrocéder une partie de leur production à leurs concurrents ${ }^{1}$ (virtual power plants). En Italie, ENEL a été obligée de rétrocéder à ses concurrents 3700 MW dans le sud de l'Italie et 150 MW en Sicile. En 2002, elle s'est également séparée de 15100 MW de capacités de production par la vente de trois de ses filiales de production : Eurogen (7 $000 \mathrm{MW})$, Elletrogen (5 $500 \mathrm{MW}$ ) et Interpower (2 $600 \mathrm{MW}$ ). En France, EDF a rétrocédé au travers d'un système d'enchères 1025 MW (736 MW en base, 289 MW en pointe) en septembre 2007. Dans le cadre de la loi NOME, une obligation lui est faite de rétrocéder $25 \%$ de sa production nucléaire à ses concurrents au prix régulé de 40€/MWh (Créti et al. [2013]). Ces cessions peuvent concerner soit des capacités physiques de production (cas de l'ENEL ou de la Californie) soit uniquement l'énergie produite, les contrats d'importation, d'achat ou encore les centrales de production restant la propriété de l'opérateur rétrocédant.

\subsection{Régulation symétrique et asymétrique : leçons de la littérature économique}

Une régulation symétrique permet de conserver l'efficacité productive et améliore les performances du marché, en particulier lorsque les asymétries de coûts entre les opérateurs sont importantes (Schankerman et Waverman [1997]). Schankerman [1996] indique qu'une régulation symétrique doit toujours être préférée à une RA excepté dans les situations pour lesquelles un comportement anticoncurrentiel a été observé ou s'il n'existe pas de moyens moins coûteux socialement pour favoriser l'émergence d'un marché concurrentiel. Une RA peut être coûteuse et inutile si les évolutions du marché sont

\footnotetext{
${ }^{1}$ En Californie, les OH devaient rétrocéder la propriété totale des centrales de production à leurs concurrents pour satisfaire aux obligations de séparation des activités. Cette mesure a contribué aux problèmes connus lors de la crise de 2003 en facilitant les pouvoirs de marché et la désincitation aux investissements.
} 
favorables à l'entrée de nouveaux concurrents et à l'émergence de nouvelles sources d'approvisionnement (Beesley [1997]). Au contraire, elle peut contribuer à un développement accéléré du marché et des facteurs favorisant la concurrence en déclenchant des investissements de la part des bénéficiaires de cette régulation (Lyon et Huang [1995]). Tous les auteurs sont cependant d'accord sur le fait qu'une RA favorise les entrées, créant des opportunités de profits indépendantes des efficacités des opérateurs. Selon Armstrong [1999], ces opportunités seraient liées à une dispense d'assurer certaines obligations (la sécurité d'approvisionnement) ou de profiter d'une part de marché imposée à l'OH, ouvrant la porte aux stratégies d'écrémage ou de reverse cherrypicking. Carsberg [1993] indique qu'une condition de succès d'une RA est de permettre l'entrée d'un nombre suffisant de concurrents pour limiter ou éviter les comportements stratégiques et collusifs qui pourraient découler de cette régulation. Une RA obligeant la vente de capacités de production par un opérateur à ses concurrents est susceptible d'engendrer des phénomènes de collusion, phénomènes qui n'étaient jusqu'alors pas observés (Ivaldi et al. [2003b]). Les marchés énergétiques sont naturellement porteurs d'incitations à la collusion (Green et Newberry [1992]), favorisées par l'intervention sur plusieurs marchés des opérateurs (Bernheim et Whinston [1990]). Généralement, la concurrence avec capacités de production symétriques conduit à une collusion soutenable (Penard [1997], Ivaldi et al. [2003a]). Conserver des niveaux d'asymétrie contribuerait à réduire cette incitation (Compte et al. [2002]). A côté de ce constat, le nombre d'entreprises agissant sur un marché peut également être un facteur aggravant la collusion (Brock et Scheinkman [1985]). Une augmentation du nombre d'acteurs sur le marché réduit ce risque d'ententes (Ivaldi et al. [2003a]).

Selon Armstrong et al. [1994], la possession d'un input n'est pas a priori une barrière à l'entrée, ou sous-jacente à un comportement anticoncurrentiel. Elle confère simplement une rente à celui qui le possède. Si ces inputs sont associés à des contrats de long terme, alors ils peuvent conduire à des mesures de RA si leur propriétaire refuse de les ouvrir alors que ses concurrents 
n'ont pas accès à l'input ou s'ils lui procurent un droit de préemption sur les fournisseurs. Les expériences empiriques de gas release montrent que ces mesures ont été adoptées dans l'attente d'un développement des infrastructures de transport et d'importation. Des investissements en capacité pour sécuriser la fourniture sur un marché, au côté de mesures complémentaires de RA, améliorent significativement le welfare (Chaton et al. [2012]). .

La littérature sur les RA met donc l'accent sur l'efficacité des entrées, des investissements et de l'industrie. Elle n'aborde pas le problème des coûts échoués irrécupérables, pouvant découler de l'introduction de cette concurrence forcée.

\subsection{Les coûts échoués irrécupérables : optimalité du}

\section{financement et impacts concurrentiels}

Les opérateurs historiques ont effectué des investissements dans un contexte protégé, avec l'obligation de service public c'est-à-dire de servir au mieux toute la demande qui s'adressait à eux. Cette situation les a conduits à effectuer des investissements basés sur les évolutions de demande qu'ils servaient en totalité. L'amortissement et le financement de ces investissements ont donc été calculés dans ce contexte. L'apparition de concurrents et du nouvel environnement concurrentiel a remis en cause ces perspectives de rentabilité. Les opérateurs historiques se sont alors retrouvés pour certains dans l'impossibilité de recouvrer certains coûts, la demande et les prix sur les marchés n'étant plus ceux anticipés lors des prises de décisions des investissements passés. Nous considérerons dans la suite de l'étude que ces investissements ont été effectués dans le secteur de la production. Ce sont par exemple des actifs de production électrique non utilisés, des contrats de ventes d'électricité dénoncés par une modification organisationnelle du marché ou encore des accords d'achats par contrats de long terme de quantités de gaz naturel (Baumol et Sidak [1995]). Ces investissements déjà entrepris deviennent alors des coûts échoués irrécupérables pour l'entreprise en place. Ces coûts 
échoués irrécupérables pèsent sur l'efficacité des opérateurs qui les subissent et doivent les financer. Ils sont susceptibles de conduire à une moindre efficacité de l'industrie en permettant l'entrée de concurrents inefficaces, qui voient alors des perspectives de profit se dessiner. Cette situation rejoint les craintes que font peser les régulations asymétriques sur les efficacités productive et allocative. Par conséquent, il peut s'avérer optimal pour le consommateur, efficace et équitable pour l'activité, que ces coûts soient pris en compte dans les systèmes de régulation qui encadrent le nouvel environnement concurrentiel. Une régulation asymétrique introduit une concurrence "forcée", entraînant des coûts échoués irrécupérables pour les opérateurs historiques. Ces derniers subissent l'obligation de rétrocéder des actifs de production (centrales électriques) ou des biens produits (électricité ou gaz) aux concurrents. Dès lors, la régulation asymétrique peut contribuer au financement des coûts échoués irrécupérables qu'elle peut engendrer. La littérature développée autour de ces questions d'optimalité de financement des coûts échoués irrécupérables concerne majoritairement le développement et la régulation des réseaux. Cependant, elle est pertinente pour analyser les coûts échoués irrécupérables dans le secteur de la production. En effet, le secteur dispose de rendements d'échelle croissants qui nécessitent que la production ait atteint une taille critique pour être rentabilisée par des prix concurrentiels. Ensuite, ces investissements échoués irrécupérables sont le fait d'une modification réglementaire mise en place par les autorités publiques et de régulation. Enfin, ces moyens de production peuvent être nécessaires pour sécuriser et maintenir la qualité de fourniture dans le système électrique (capacités de réserves ou de back-up).

Cette littérature développe un argumentaire sur la pertinence de financer ou non les coûts échoués irrécupérables, qu'ils soient observés dans les secteurs de la production ou des réseaux. Joskow [2008a] indique que les gains de court terme réalisés grâce au non-financement des coûts échoués irrécupérables peuvent être largement compensés par les pertes futures en termes de qualité de service. Du point de vue des consommateurs, ce second point 
s'avère important, parfois même plus important que les gains liés à des diminutions de prix. Berry [2000] étudie le financement de ces coûts à travers une tarification d'accès au réseau de second rang. Il suppose que les consommateurs sont différenciés notamment quant aux incitations à quitter l'OH pour le marché concurrentiel (mesurées par "l'élasticité de sortie"). Les consommateurs ayant une élasticité "de sortie" faible financeront les coûts échoués irrécupérables en faveur des consommateurs à élasticité "de sortie" forte. Il note également que les tarifs d'accès aux réseaux pour des consommateurs ayant choisi de quitter l'OH sont proportionnels à l'élasticité "de sortie" de ces consommateurs. Cette élasticité "de sortie" peut également impacter les systèmes de production en réintroduisant des rendements d'échelle croissants dans le secteur, impactant leur financement comme le montrent les travaux sur les mécanismes de capacité ou sur l'effet économique bien connu du missing money.

Kolbe et Tye [1996] notent que les investisseurs ne peuvent pas être compensés pour les coûts échoués irrécupérables par le coût du capital ou le taux de rendement déterminé par la régulation ex ante. Une prime de risque peut s'avérer nécessaire, conduisant à une augmentation du coût du capital ou du taux de rendement, augmentation qui compense l'écart entre les revenus réalisés et espérés de l'activité. Joskow [1996] montre que le recouvrement des coûts échoués irrécupérables n'est pas synonyme de distorsion de concurrence ni de faveurs envers des opérateurs inefficaces économiquement. Ce recouvrement nécessite que ces coûts soient clairement définis et estimés. Il doit de plus se réaliser auprès de tous les consommateurs, indépendamment de leur fournisseur. Lesser et Ainspan [1996] montrent qu'une approche par un système d'enchères est le moyen le plus efficace d'évaluer et de rémunérer les coûts échoués irrécupérables. Ils notent toutefois qu'il est nécessaire pour les consommateurs de se couvrir contre les risques prix, la rémunération engendrée pouvant se traduire par des variations de prix significatives. Tye et Graves [1996] notent que ce recouvrement permet à chacun des acteurs de se concurrencer en fonction de sa réelle efficacité. Bien que 
quelquefois synonyme de distorsion de concurrence, ils notent que la neutralité concurrentielle est souvent vérifiée car le coût incrémental des opérateurs est majoré équitablement de la prime de financement des coûts échoués irrécupérables. Des modes de financement tels que la collecte du financement par une tierce partie auprès de tous les acteurs ou la collecte par l'OH auprès des entrants vérifient cette neutralité. Maloney et Sauer [1998] sont plus réservés quant à la nécessité de financer ex post des investissements (notamment de production) qui s'avèrent être des coûts échoués irrécupérables. En effet, les autorités de régulation ne doivent pas internaliser les risques liés aux investissements, risques habituellement supportés par les investisseurs contre un taux de rendement incluant une prime de risque. Que les investissements ex ante dans la production soient efficaces ou non, le consommateur n'a pas à subir le risque de mauvaises anticipations sur les évolutions réglementaires ou concurrentielles de la part des investisseurs. Les régulateurs se doivent de conserver les incitations qu'aurait donnée l'existence d'un marché pour atteindre l'efficacité allocative et productive, et non de subventionner les investissements non compétitifs. Selon Beard et al. [2003], il n'est pas optimal collectivement pour un régulateur de permettre le recouvrement total des coûts échoués irrécupérables. En effet, le transfert entre entreprise régulée et consommateur est coûteux, d'autant plus lorsque le revenu autorisé est croissant avec les coûts échoués irrécupérables à financer.

\section{Régulation asymétrique : un mécanisme ré- gulé de financement des coûts échoués ir- récupérables}

Le financement des coûts échoués irrécupérables en présence de régulation asymétrique complémente les littératures présentées jusqu'alors. En effet, le régulateur dispose d'un nouvel outils, au travers du couple "prix de rétrocession et quantités rétrocédées" caractérisant la RA, pour internaliser ce 
financement. Pour définir cette politique de régulation optimale, nous utilisons un modèle simple de duopole à la Cournot $^{2}$ présenté dans d'autres travaux avec ou sans contraintes sur les capacités de production (Clastres [2005], Clastres et David [2009], Chaton et al. [2008]).

\subsection{Régulation asymétrique et concurrence "à la Cour- not" avec une seule source d'approvisionnement}

Le marché se composera d'un $\mathrm{OH}$ qui détient les capacités existantes de production d'inputs et un concurrent qui ne dispose pas d'accès à cet in$p u t^{3}$. Chacun intervient sur le marché final (ou marché aval) ainsi que sur un marché intermédiaire (ou d'approvisionnement). Le concurrent et l'OH se concurrencent à la Cournot sur le marché final, vendant respectivement $q_{e}$ et $q_{o}$. La demande $p(q)$ qui s'observe sur le marché final sera supposée linéaire, $p(q)=1-q$, avec $q=q_{e}+q_{o}$. Sur le marché final, les profits des deux opérateurs sont égaux à $\Pi_{i}^{f}=p(q) q_{i}, i=\{e, o\}$, les coûts de distribution et de fourniture étant supposés symétriques et normalisés à 0 pour les deux opérateurs. Le surplus du consommateur sera donné par la formule usuelle $S_{c}=\int_{0}^{q} p(t) d t-p(q) q$ telle que $S_{c}^{\prime}(q)=p(q)$. L'OH et son concurrent agissent en information parfaite et complète. L'OH dispose d'actifs de production en quantités $K_{o}>0$ au coût unitaire $u>0$. Nous supposerons que ces capacités de production d'inputs $K_{o}$ sont suffisamment importantes pour permettre à l'OH de toujours produire et jouer sa stratégie de meilleure réponse $q_{o}$ et ce même après le processus de rétrocession d'inputs à son concurrent ${ }^{4}$. Ces conditions d'approvisionnement $u K_{o}$ pourront et seront interprétées comme

\footnotetext{
${ }^{2}$ Pour une analyse sur l'utilisation d'un modèle de Cournot dans les marchés énergétiques, se référer à Breton et Zaccour (2001) ou Bremberger et al. (2012).

${ }^{3}$ Les actifs de production concerneront ici les centrales de production électriques ou les contrats d'approvisionnement gazier de long terme du type Take or Pay. L'input sera ici l'électricité produite ou la molécule de gaz. Le manque d'accès à ces inputs pour les concurrents potentiels à l' $\mathrm{OH}$ entraîne le recours à une régulation asymétrique.

${ }^{4}$ Pour une résolution incluant une contrainte d'approvisionnement pour l'OH, se référer à Clastres et David [2009].
} 
l'efficacité de l'OH. En effet, les capacités de production $K_{o}$ étant considérées comme importantes, l'OH sera efficace si le coût unitaire $u$ de ces capacités a été bien négocié, c'est-à-dire lorsque $u$ est suffisamment faible pour que les conditions d'approvisionnement $u K_{o}$ soient suffisamment efficaces. Des conditions d'approvisionnement très inefficaces, $u K_{o}>\frac{1}{4}$, ne permettent pas à un monopole de réaliser des profits non négatifs ${ }^{5}$. Les structures de monopole privée ou de monopole public réglementé nous permettent donc de borner ces conditions d'approvisionnement : $\left.\left.u K_{o} \in\right] 0, \frac{1}{4}\right]$.

Le régulateur impose à l'OH une RA : rétrocéder une partie $\alpha \in] 0,1]$ de ses approvisionnements $K_{o}$ au prix régulé $r \geq 0$. Le couple $(r, \alpha)$ caractérise la RA et constitue les leviers concurrentiels et de financements des coûts échoués irrécupérables pour le régulateur. Le concurrent ne s'approvisionne qu'auprès de l'OH grâce aux rétrocessions de capacités pour une quantité $K_{e}$. Le niveau des approvisionnements de l'OH sont tels que seul le concurrent subit une contrainte sur son approvisionnement ${ }^{6}: K_{e} \leq \alpha K_{o}$. Sur le marché intermédiaire, les profits des opérateurs sont $\Pi_{e}=p(q) q_{e}-r K_{e}$ pour le concurrent et $\Pi_{o}=p(q) q_{o}-u K_{o}+r K_{e}$ pour l'Opérateur Historique. La fonction de bien être-social $W$ sera définie comme la somme de ces profits et du surplus du consommateur, c'est à dire $W=\Pi_{e}+\Pi_{o}+S_{c}=p(q) q-u K_{o}+$ $\frac{1}{2} q^{2}$. Le déroulement du jeu sera le suivant :

- Etape 1 : Le régulateur choisit sa politique de régulation $(\alpha, r)$ de manière à maximiser le bien-être collectif. La politique de régulation est ici endogénéisée à la manière de plusieurs travaux (Clastres [2005], Chaton et al. [2008], Clastres et David [2009]).

- Etape 2 : Le concurrent, connaissant $\alpha$ et $r$, choisit son niveau d'approvisionnement $K_{e} \leq \alpha K_{o}$ qui maximise son profit $\Pi_{e}$.

\footnotetext{
${ }^{5}$ Les équilibres de monopole privé $\left(q^{m *}, p^{m *}\right)=\left(\frac{1}{2}, \frac{1}{2}\right)$, avec comme profit $\Pi_{o}^{m *}\left(q^{m *}, p^{m *}\right)=\frac{1}{4}-u K_{o}$, ou public réglementé $\left(q^{R B *}, p^{R B *}\right)=\left(\frac{\sqrt{1-4 u K_{o}}+1}{2}, \frac{1-\sqrt{1-4 u K_{o}}}{2}\right)$ nous conduisent à borner les valeurs d'approvisionnement de l'OH qui sont telles que $\left.\left.u K_{o} \in\right] 0, \frac{1}{4}\right]$.

${ }^{6}$ Pour une analyse avec deux opérateurs contraints, se référer à Breton et Zaccour (2001) ou Clastres et David (2009).
} 
- Etape 3 : L'OH et son concurrent se livrent une concurrence "à la Cournot" sur le marché final. Le concurrent subit sa contrainte d'approvisionnement $K_{e}$ négociée à la seconde étape.

\subsection{Les équilibres en sous-jeux}

\subsubsection{Les équilibres sur le marché aval}

Les deux opérateurs maximisent leurs profits respectifs sur le marché aval. Le concurrent maximise son profit sous contrainte que la quantité trouvée respecte sa contrainte d'approvisionnement : $\operatorname{Max}_{e} \Pi_{e}^{f} s / c q_{e} \leq K_{e}$. L'OH maximise son profit sans subir de contrainte : $\operatorname{qax}_{q_{o}} \Pi_{o}^{f}$.

Le problème d'optimisation nous conduit à résoudre le système suivant :

$$
\left\{\begin{array}{c}
L_{e}^{f}=p(q) q_{e}+\lambda_{e}\left(q_{e}-K_{e}\right) \\
\Pi_{o}^{f}=p(q) q_{o} \quad\left(\lambda_{e}\right)
\end{array}\right.
$$

Lorsque $K_{e}>\frac{1}{3}$, l'équilibre est $\left\{\begin{array}{l}q_{o}^{*}=\frac{1}{3} \\ q_{e}^{*}=\frac{1}{3}\end{array}\right.$

Lorsque $K_{e} \leq \frac{1}{3}$, l'équilibre est $\left\{\begin{array}{c}q_{o}^{c *}=\frac{1}{2}-\frac{1}{2} K_{e} \\ q_{e}^{c *}=K_{e} \\ \lambda_{e}^{c *}=-\frac{3}{2} K_{e}+\frac{1}{2}\end{array}\right.$

L'interprétation de cet équilibre en sous jeu indique intuitivement que lorsque le concurrent dispose de capacité d'approvisionnement suffisante $K_{e}$, alors sa stratégie n'est pas contrainte et l'équilibre de Cournot-Nash noncontraint peut être joué. Sinon, il est contraint par ses approvisionnements et offre $q_{e}^{* c}=K_{e}$. Dans ces deux situations, l'OH peut lui toujours jouer sa stratégie de meilleure réponse sans contrainte.

\subsubsection{Les équilibres sur le marché des approvisionnements}

L'OH subit les coûts de ses investissements passés $\left(u K_{o}\right)$ et bénéficie des gains de la rétrocession de ses capacités $\left(r K_{e}\right)$ et liés à ses ventes sur le marché aval $\left(p(q) q_{o}\right)$. Son profit sera $\Pi_{o}^{A}=p(q) q_{o}-u K_{o}+r K_{e}$. Il ne joue pas 
à cette étape, $K_{o}$ étant déterminé. Le concurrent, lui, a la possibilité d'enlever toute ou partie de la quantité $\alpha K_{o}$ que l'OH est obligé de rétrocéder. Il va donc maximiser son profit compte tenu de la contrainte qui pèse sur lui :

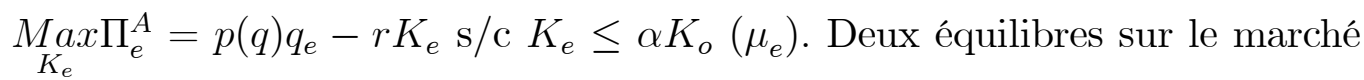
des approvisionnements résultent de cette résolution.

Si $K_{e}<\alpha K_{o}$, alors $\mu_{e}=0$ et la quantités d'approvisionnement que va acheter le concurrent est $K_{e}^{*}=\frac{1}{2}-r$. Lorsque $\alpha \geq \frac{1-2 r}{2 K_{o}}$ et $\frac{1}{6} \leq r<\frac{1}{2}$, l'équilibre sur le marché final est alors $\left\{\begin{array}{c}q_{o}^{c *}=\frac{1}{4}+\frac{1}{2} r \\ q_{e}^{c *}=\frac{1}{2}-r \\ K_{e}^{*}=\frac{1}{2}-r \\ \lambda_{e}^{c *}=-\frac{1}{4}+\frac{3}{2} r\end{array}\right.$

Si $K_{e}=\alpha K_{o}$, alors $\mu_{e}^{*}=-\alpha K_{o}+\frac{1}{2}-r$ et la quantité optimale d'approvisionnement est donc $K_{e}^{*}=\alpha K_{o}=\frac{1}{2}-r$. Lorsque $\alpha \leq \frac{1-2 r}{2 K_{o}}$, alors l'équilibre sur le marché final est $\left\{\begin{array}{c}q_{o}^{c *}=\frac{1}{2}-\frac{1}{2} \alpha K_{o} \\ q_{e}^{c *}=\alpha K_{o} \\ K_{e}^{*}=\alpha K_{o} \\ \lambda_{e}^{c *}=-\frac{3}{2} \alpha K_{o}+\frac{1}{2} \\ \mu_{e}^{*}=-\alpha K_{o}+\frac{1}{2}-r\end{array}\right.$

Le premier équilibre indique que la rétrocession de capacités ordonnée par le régulateur est en quantité suffisamment importante pour que les deux acteurs puissent jouer leur stratégie d'équilibre de Cournot sur le marché aval sans subir de contraintes sur leurs approvisionnements. Le second équilibre contraint le concurrent dans ses approvisionnements, la proportion rétrocédée étant trop faible.

\subsection{L'équilibre du jeu : choix de la politique de régu- lation par le régulateur}

En fixant sa politique de régulation asymétrique, le régulateur cherche à maximiser le bien-être collectif. La fonction de welfare est une fonction croissante des quantités offertes sur le marché. Par conséquent, le régula- 
teur cherche à maximiser l'offre des opérateurs sur le marché. En effet, si le concurrent subit une contrainte, cela implique qu'il aurait souhaité offrir davantage de biens sur le marché et donc que la contrainte réduit l'offre globale (Clastres et David [2009]). Par conséquent, le régulateur a intérêt de choisir une proportion rétrocédée telle que la stratégie $q_{e}^{c *}=\frac{1}{2}-r$ puisse être jouée (dans la limite de $q_{e}^{c *}=\frac{1}{3}$ qui est l'équilibre de Nash-Cournot non contraint). Pour cela, la proportion optimale rétrocédée $\alpha^{*}$ doit satisfaire la condition $\alpha^{*} \geq \frac{1-2 r}{2 K_{o}}$. Le régulateur peut déterminer toute proportion rétrocédée respectant cette condition. La proportion minimale qu'il choisira sera telle que $\alpha^{*} K_{o}=q_{e}^{c *} \Leftrightarrow \alpha^{*}=\frac{1-2 r}{2 K_{o}}$. Cette quantité permet à chacun de jouer sa stratégie de meilleure réponse sans contrainte.

Le régulateur se situe sur la partie décroissante du welfare pour la détermination du prix $r^{*}$. Aussi, il va rechercher le prix $r^{*}$ minimum, c'est-à-dire fixer un prix $r^{*}=\frac{1}{6}$ pour satisfaire les équilibres en sous-jeux. Nous remarquons que pour ce prix, $q_{e}^{c *}=\frac{1}{3}$ et $\alpha^{*}=\frac{1}{3 K_{o}}$. L'équilibre de Cournot sans contrainte est joué sur le marché aval. Cela signifie que fixer un prix inférieur à $r^{s}$ est possible mais n'a pas pour autre impact que de créer des transferts de profits entre l'OH et le concurrent.

L'équilibre du jeu est donc donné par $\left\{\begin{array}{c}q_{e}^{*}=\frac{1}{3} \\ q_{o}^{*}=\frac{1}{3} \\ K_{e}^{*}=\frac{1}{3} \quad \text { (Eq.6) } \\ \alpha^{*}=\frac{1}{3 K_{o}} \\ r^{*}=\frac{1}{6}\end{array}\right.$

Conclusion 1 Le régulateur adapte sa politique de régulation de manière à ne pas contraindre les deux opérateurs. En établissant sa politique de régulation telle que $\alpha^{*}=\frac{1}{3 K_{o}}$ et $r^{*}=\frac{1}{6}$, le régulateur converge vers l'équilibre non contraint $\left(\frac{1}{3}, \frac{1}{3}\right)$ pour lequel le concurrent achète toutes les quantités rétrocédées et maximise le bien-être collectif.

Conformément à ce qu'évoque la littérature sur la RA, le concurrent est actif sur le marché. En effet, la RA lui permet de réaliser une activité profitable; il réalise des profits toujours positifs. Le régulateur, en déterminant sa 
politique de régulation, réalise des transferts de profit entre les deux acteurs (Breton et Zaccour [2001]). En revanche, selon les niveaux de coût ou d'approvisionnement de l'OH, la concurrence peut faire encourir à l'OH des coûts échoués irrécupérables. Des pertes peuvent apparaître en présence de ventes totales de l'OH trop faibles par rapport à ses capacités de production ou dans des cas de prix de rétrocession insuffisamment rémunérateur par rapport aux coûts des actifs de production.

\subsection{Le régulateur et le financement des coûts échoués irrécupérables : les prix Ramsey-Boîteux}

La RA modifie le contexte concurrentiel et réglementaire dans lequel l'OH intervient. Nous sommes ici dans le cadre théorique de l'apparition des coûts échoués irrécupérables. Le régulateur dispose de la possibilité d'adapter sa politique de RA pour s'assurer que le profit de l'OH est non négatif. Ainsi, il ne le défavorise pas devant ses concurrents et évite sa sortie du marché ${ }^{7}$. Le régulateur laisse l'OH actif sur le marché, tout en facilitant l'accès à la ressource pour le concurrent. La proportion rétrocédée est déterminée comme précédemment, c'est-à-dire de telle sorte que le concurrent puisse jouer sa meilleure réponse, $q_{e}^{S B *}=\alpha^{S B *} K_{o}=\frac{1}{2}-r^{S B *}$. Le prix de rétrocession est tel qu'il permettra de maximiser le bien-être contraint. Le programme d'optimisation $\mathrm{s}^{\prime e ́ c r i t}{ }^{8}$ :

$$
\left\{\begin{aligned}
r^{S B *}= & \arg \left\{\underset{r}{\operatorname{Max} W}=\left(1-q^{*}\right) q^{*}-u K_{o}+\frac{1}{2}\left(q^{*}\right)^{2}\right\} \\
& \text { s/c } p\left(q^{*}\right) q_{o}^{*}-u K_{o}+r q_{e}^{*} \geq 0 \quad\left(\mu_{o}\right)
\end{aligned}\right.
$$

avec $q^{*}=q_{o}^{*}+q_{e}^{*}$. La liberté de la contrainte nous renvoie au cas précédent avec un profit pour l'OH $\Pi_{o}^{*}=\frac{1}{6}-u K_{o}$ positif pour $u K_{o} \in\left[0, \frac{1}{6}\right]$. Le profit de duopole suffit pour financer les coûts échoués irrécupérables de l'OH.

\footnotetext{
${ }^{7}$ Le risque de sortie du marché suite à une rétrocession de capacités a été empiriquement observé sur lors de la crise du marché électrique californien de 2003.

${ }^{8}$ Les variables $r$ et $\alpha$ qui maximiseront le welfare doivent être non-négatives. Par soucis de simplicité dans la résolution des conditions de premier ordre, nous vérifierons ex-post pour tous les équilibres que les variables solutions sont non-négatives.
} 
Avec saturation de la contrainte, le seul prix d'équilibre qui soit possible est $r^{S B *}=\frac{1}{2}-\frac{1}{3} \sqrt{3-12 u K_{o}}$, avec $u K_{o}>\frac{1}{6}$ et $\mu_{o}^{S B *}=\frac{1}{6} \frac{\sqrt{3}-\sqrt{1-4 u K_{o}}}{\sqrt{1-4 u K_{o}}}$, variable duale positive pour $u K_{o}<\frac{1}{4}$.

Pour $\left.\left.u K_{o} \in\right] \frac{1}{6}, \frac{1}{4}\right]$, l'équilibre du jeu est

$$
\left\{\begin{array}{c}
q_{o}^{S B *}=\frac{1}{2}-\frac{1}{6} \sqrt{3-12 u K_{o}} \\
q_{e}^{S B *}=\frac{1}{3} \sqrt{3-12 u K_{o}} \\
r^{S B *}=\frac{1}{2}-\frac{1}{3} \sqrt{3-12 u K_{o}} \\
\alpha^{S B *}=\frac{1}{3} \frac{\sqrt{3-12 u K_{o}}}{K_{o}}
\end{array}\right.
$$

Le régulateur peut ici financer les coûts échoués irrécupérables grâce uniquement à sa politique de régulation. Il détermine le prix de rétrocession en fonction des conditions d'approvisionnement de l'OH ${ }^{9}$. Plus ces conditions sont dégradées, plus le prix de rétrocession sera élevé, aux dépends des quantités achetées et vendues sur le marché final par le concurrent.

Proposition 1 Pour un OH efficace, $u K_{o} \in\left[0, \frac{1}{6}\right]$, le prix de rétrocession d'équilibre $r^{*}=\frac{1}{6}$ maximise le welfare et permet à l'OH de réaliser des profits positifs. Le profit de duopole suffit pour financer les coûts échoués irrécupérables. Pour un $O H$ inefficace, $u K_{o} \in\left[\frac{1}{6}, \frac{1}{4}\right]$, le prix de rétrocession d'équilibre $r^{S B *}$ permet de maximiser le welfare en finançant les coûts échoués irrécupérables.

\section{Preuve. Annexe 1.}

Les transferts de surplus entre les agents vont être déterminés par l'efficacité de l'OH, efficacité sous-jacente aux politiques de régulation asymétrique mises en place par le régulateur. La meilleure allocation des différents surplus est déterminée pour un $\mathrm{OH}$ efficace, c'est-à-dire lorsque $u K_{o} \leq \frac{1}{6}$. Lorsque l'efficacité de l'OH se dégrade, $u K_{o}>\frac{1}{6}$, alors le régulateur, pour conserver

\footnotetext{
${ }^{9}$ Ce prix tient également compte de l'équilibre $r^{*}=\frac{1}{6}$ qui impacte le niveau de profit de l'OH. En effet, le prix $r^{*}=\frac{1}{6}$ permet de minimiser pour l'OH la zone de pertes liée aux coûts échoués irrécupérables. Choisir un prix inférieur à $\frac{1}{6}$ augmentera cette zone de perte, le régulateur se devant d'adapter sa RA en augmentant le prix $r^{S B *}$.
} 
les deux opérateurs sur le marché, adapte sa régulation en fixant un prix de rétrocession $r^{S B *}$ afin de financer les coûts échoués irrécupérables apparus suite à l'introduction de la concurrence forcée. Le profit de l'OH est alors nul, le surplus du consommateur se réduit car le prix sur le marché augmente et le profit du concurrent est moins important car la baisse des quantités vendues associée à une augmentation de ses coûts d'approvisionnement ne sont pas compensées par une augmentation des prix de vente sur le marché final. Par conséquent, des transferts de surplus sont mis en place du concurrent et du consommateur vers l'OH. Tous les surplus sont dégradés ce qui diminue le welfare global et la satisfaction de chaque agent.

Une analyse plus dynamique peut découler des résultats des (Eq.6) et (Eq.8) en termes d'incitations aux investissements ${ }^{10}$. Le concurrent réalise des profits toujours positifs à la suite de la RA. Ce résultat conduit à deux stratégies possibles pour le concurrent. La première est de s'assurer que la période de concurrence forcée dure sur une longue période pour accumuler les résultats positifs sans avoir à investir pour satisfaire sa demande. Cette stratégie suppose que les régulateurs reconduisent les périodes de RA. La seconde est au contraire de tirer profit de ces gains positifs pour développer ses propres approvisionnements ou infrastructures de production pour pérenniser la satisfaction de sa demande, demande acquise grâce au processus de concurrence forcée (Lyon et Huang [1995]). S'intégrer vers l'amont lui permet également de mieux gérer les risques liés à ses conditions d'approvisionnement ou de production en maîtrisant une partie de la chaine des coûts et d'éviter le risque lié à la régulation. Ces stratégies d'intégration verticale ont été observées sur plusieurs marchés qui ont implémentés ces processus de RA (Espagne, Allemagne, France). En effet, l'objectif d'une RA est de favoriser la concurrence durant une durée limitée (généralement de 5 ans). A la suite de cette période, les concurrents se doivent de pouvoir assurer la

\footnotetext{
${ }^{10}$ Pour une analyse des incitations aux investissements dans le cadre d'une RA, se référer à Smeers [1997], Schankerman [1996], Schankerman et Waverman [1997] ou Lyon et Huang [1995].
} 
fourniture de leur demande par des actions propres. Le régulateur n'intervient alors que pour éviter les pouvoirs de marché (conjointement avec les autorités de concurrence) et réguler les activités de réseaux. La rétrocession de moyens de production n'est plus assurée. L'existence de ces horizons finis couplée aux bénéfices réalisés grâce à la RA ont incité les concurrents à développer leurs infrastructures.

L'OH subit également des incitations controversées sous une RA. Il réalise des bénéfices lorsque d'une part les coûts échoués irrécupérables sont financés et d'autres part s'il est suffisamment efficace. En ce sens, il est incité à développer des infrastructures de production les plus efficaces possibles pour conserver une rente. Cependant, il peut se retrouver dans des situations pour lesquelles les rentes sont redistribuées aux consommateurs et aux concurrents, le laissant avec un revenu couvrant au plus juste ses coûts. Dès lors, son incitation à développer son offre peut s'avérer réduite. Ici encore, l'horizon fini de la RA peut l'inciter à investir efficacement dans l'optique de récupérer une partie des actifs temporairement rétrocédés aux concurrents. Le développement du marché concurrentiel conforte cette intuition. L'OH étant désormais en concurrence sur son marché (Smeers, 1997), il est incité à investir le plus efficacement possible pour conserver d'une part sa clientèle (qualité de fourniture, des services) et d'autres part sa compétitivité face à des concurrents pouvant bénéficier plus rapidement des nouvelles technologies (Perrucci et Cimatoribus [1997]). Cependant, les mesures de RA, bien qu'ayant favorisées des entrées de concurrents, n'ont pas résolu le manque d'incitation aux investissements sur les marchés, particulièrement électriques. En effet, des mécanismes incitatifs complémentaires aux seuls profits positifs sont jugés nécessaires pour internaliser les risques de volatilité des prix, d'incertitude sur la demande ou de missing money (Cramton et Stoft [2005], Joskow [2008b]). 


\section{L'introduction d'une source alternative d'ap- provisionnement}

Nous introduisons désormais la possibilité pour le concurrent de s'approvisionner auprès d'une seconde source d'approvisionnement. Cette nouvelle source est disponible en quantités $K$ au coût unitaire $s \geq 0$. Le concurrent achète $K_{s}$ auprès de cette nouvelle source, $K_{s} \leq K$ en sus de ses achats de capacités rétrocédées, $K_{e}^{s} \leq \alpha^{s} K_{o}$.

La source alternative d'approvisionnement peut avoir un effet positif sur le welfare en permettant aux deux opérateurs de maximiser leur offre sur le marché final en gagnant en efficacité. Intuitivement, si la source alternative est compétitive et en quantités suffisantes, la mesure de RA n'est pas nécessaire, le concurrent allant s'approvisionner uniquement auprès de la source alternative pour concurrencer l'OH. En revanche, si cette source est compétitive mais en quantité insuffisante, ou si cette source est non compétitive, la RA peut permettre au concurrent de diminuer la contrainte d'approvisionnement qui pèse sur lui.

\subsection{Les équilibres en sous-jeux}

\subsubsection{Les équilibres sur le marché aval}

Le concurrent maximise son profit sous sa contrainte d'approvisionnement :

$\underset{q_{e}^{s}}{\operatorname{Max} \Pi_{e}^{f s} \quad s / c} q_{e}^{s} \leq K_{e}^{s}+K_{s}\left(\lambda_{e}^{s}\right)$. L'OH maximise son profit $\Pi_{o}^{f s}$ sans subir de contrainte.

Lorsque $\left(K_{e}^{s}+K_{s}\right)>\frac{1}{3}$, les niveaux d'approvisionnements sont suffisamment élevés et compétitifs pour chacun. L'équilibre est celui de Nash-Cournot non contraint $\left\{\begin{array}{l}q_{e}^{s *}=\frac{1}{3} \\ q_{o}^{s *}=\frac{1}{3}\end{array} \quad(\right.$ Eq.9)

En revanche, si $\left(K_{e}^{s}+K_{s}\right) \leq \frac{1}{3}$, alors l'entrant dispose de sources d'approvisionnement trop faibles. Il vend sur le marché toutes les quantités dis- 
ponibles auprès des deux sources d'approvisionnement. L'équilibre est alors

$$
\left\{\begin{array}{c}
q_{e}^{s c}=K_{e}^{s}+K_{s} \\
q_{o}^{s c}=\frac{1}{2}-\frac{1}{2}\left(K_{e}^{s}+K_{s}\right) \\
\lambda_{e}^{s c}=-\frac{3}{2}\left(K_{e}^{s}+K_{s}\right)+\frac{1}{2}
\end{array}\right.
$$

\subsubsection{Le choix optimal des approvisionnements}

Le concurrent peut acheter $K_{e}^{s} \leq \alpha^{s} K_{o}$ (RA) et $K_{s} \leq K$ (source alternative d'approvisionnement). Il choisit son niveau d'approvisionnement en maximisant son profit par rapport à $K_{e}^{s}$ et $K_{s}$ :

$$
\left\{\begin{array}{c}
\underset{\left\{K_{e}^{s}, K_{s}\right\}}{\operatorname{Max}} \prod_{e}^{s}=p^{s}\left(q^{s}\right) q_{e}^{s}-r^{s} K_{e}^{s}-s K_{s} \\
s / c \begin{cases}K_{e}^{s} \leq \alpha^{s} K_{o} & \left(\mu_{e}^{s}\right) \\
K_{s} \leq K & \left(\mu_{s}^{s}\right)\end{cases}
\end{array}\right.
$$

Les deux contraintes saturées nous conduisent à un équilibre

$$
\left\{\begin{array} { c } 
{ q _ { e } ^ { s c * } = \alpha ^ { s } K _ { o } + K } \\
{ q _ { o } ^ { s c * } = \frac { 1 } { 2 } - \frac { 1 } { 2 } ( \alpha ^ { s } K _ { o } + K ) }
\end{array} \quad \left(\text { Eq.12) avec } \left\{\begin{array}{c}
\left(\alpha^{s} K_{o}+K\right)<\frac{1}{2}-r^{s} \\
\left(\alpha^{s} K_{o}+K\right) \leq \frac{1}{3} \\
\left(\alpha^{s} K_{o}+K\right)<\frac{1}{2}-s
\end{array} .\right.\right.\right.
$$

Les autres situations d'équilibre apparaissent lorqu'une ou plusieurs de ces contraintes sont libres et peuvent être regroupées selon la hiérarchisation des deux prix d'approvisionnement $r^{s}$ et $s^{11}$.

Lorsque $r^{s} \geq s$, le concurrent achète toute la capacité alternative $K_{s}^{*}=K$ et le reste grâce à la RA si $r^{s} \in\left[\frac{1}{6}, \frac{1}{2}\right]$. L'équilibre sera

$$
\left\{\begin{array}{c}
q_{e 2}^{s *}=\frac{1}{2}-r^{s} \\
q_{o 2}^{s *}=\frac{1}{4}+\frac{1}{2} r^{s} \\
K_{e 2}^{s *}=\frac{1}{2}-K-r^{s} \\
K_{s 2}^{*}=K \\
\lambda_{e 2}^{s c *}=-\frac{1}{4}+\frac{3}{2} r^{s} \\
\mu_{s 2}^{s *}=-s+r^{s}
\end{array}\right.
$$

\footnotetext{
${ }^{11}$ Le cas $r^{s}=s$ est inclus dans ces deux situations d'équilibre. En effet, le concurrent pourra toujours jouer sa stratégie de meilleure réponse $q_{e}^{s *}=\frac{1}{2}-r^{s}=\frac{1}{2}-s$ en saturant l'une de ses sources d'approvisionnement ou en réalisant toute combinaison linéaire entre ses deux sources d'approvisionnement.
} 
Cet équilibre impose que $K_{e}^{s}=\frac{1}{2}-K-r^{s} \geq 0 \Leftrightarrow \frac{1}{2}-r^{s} \geq K$ avec $r^{s} \geq s$. Si $\frac{1}{2}-s>K \geq \frac{1}{2}-r^{s}$, le concurrent achète $K_{s 1}^{*}=K$ et aucune quantité auprès de la RA. Il vend alors $q_{e 1}^{s c *}=K$ sur le marché. En effet, il souhaiterait pouvoir acquérir la quantité $K_{s 1}^{*}=\frac{1}{2}-s$, équilibre obtenu lorsque seulement la source alternative est accessible à un faible coût ${ }^{12}$, mais le niveau d'approvisionnement alternatif disponible $K$ ne le lui permet pas. La RA ne sert donc pas à approvisionner le concurrent qui préfère acheter les quantités $K$ auprès de la source alternative. La question de la pertinence de la RA se pose, le prix de rétrocession étant trop élevé pour qu'il intègre à ses achats une part de quantités rétrocédées. Lorsque $K \geq \frac{1}{2}-s$, le concurrent achète $K_{s 1}^{*}=\frac{1}{2}-s$ ce qui lui permet de jouer sa stratégie de meilleure réponse $q_{e 1}^{s *}$ sur le marché aval sans subir de contrainte d'approvisionnement ${ }^{13}$. Ici encore, la pertinence de mettre une RA lorsque $K \geq \frac{1}{2}-s$ se pose, aucune quantité rétrocédée n'étant acheté par le concurrent.

Lorsque la source alternative d'approvisionnement est moins compétitive que la rétrocession, $s \geq r^{s}$, alors le concurrent adopte une stratégie inverse à la précédente. Il achète toute la capacité rétrocédée et le reste auprès de l'autre source d'approvisionnement. L'équilibre sera donc

$$
\left\{\begin{array}{c}
q_{e 3}^{s *}=\frac{1}{2}-s \\
q_{o 3}^{s *}=\frac{1}{4}+\frac{1}{2} s \\
K_{e 3}^{s *}=\alpha^{s} K_{o} \\
K_{s 3}^{*}=\frac{1}{2}-\alpha^{s} K_{o}-s \\
\lambda_{e 3}^{s c *}=-\frac{1}{4}+\frac{3}{2} s \\
\mu_{e 3}^{s *}=s-r^{s}
\end{array}\right.
$$

Cet équilibre existe si $K_{s}=\frac{1}{2}-\alpha^{s} K_{o}-s \geq 0 \Leftrightarrow \frac{1}{2}-s \geq \alpha^{s} K_{o}$ avec $s \geq r^{s}$. Si $\alpha^{s} K_{o} \geq \frac{1}{2}-s$ avec $s \geq r^{s}$, alors la source alternative d'approvisionnement

\footnotetext{
${ }^{12} \mathrm{La}$ résolution du jeu lorsque le concurrent n'a accès qu'à la source alternative nous conduit à deux équilibres : $\left(q_{e}^{s *}, q_{o}^{s *}\right)=\left(K ; \frac{1}{2}-\frac{1}{2} K\right)$ lorsque $K \leq \frac{1}{2}-s$ et $\left(q_{e}^{s *}, q_{o}^{s *}\right)=$ $\left(\frac{1}{2}-s ; \frac{1}{2} s+\frac{1}{4}\right)$ lorsque $K \geq \frac{1}{2}-s$. La démonstration est disponible sur demande.

${ }^{13} \mathrm{Ces}$ deux équilibres sont bornés supérieurement par l'équilibre de Cournot non contraint sur le marché aval, $\left(q_{e}^{*}, q_{o}^{*}\right)=\left(\frac{1}{3}, \frac{1}{3}\right)$ et existe donc lorsque $K \leq \frac{1}{3}$ ou $K_{s 1}^{*} \leq \frac{1}{3}$. Lorsque ces deux valeurs sont supérieures à $\frac{1}{3}$, alors l'équilibre est $\left(q_{e}^{*}, q_{o}^{*}\right)=\left(\frac{1}{3}, \frac{1}{3}\right)$. La démonstration est disponible sur demande.
} 
ne joue plus aucun rôle dans les achats d'input du concurrent, le prix d'accès $s$ à cette ressource étant trop élevé. L'analyse permet à nouveau de scinder ce cas en deux situations d'équilibre ${ }^{14}$. Lorsque $\frac{1}{2}-r^{s} \geq \alpha^{s} K_{o} \geq \frac{1}{2}-s$ avec $s \geq r^{s}$, alors le concurrent achète la totalité des quantités rétrocédées $K_{e 3}^{s *}=\alpha^{s} K_{o}$ qu'il revend sur le marché aval, le prix d'achat $r^{s}$ étant plus faible que celui de la source alternative. Lorsque $\alpha^{s} K_{o} \geq \frac{1}{2}-r^{s}$, alors le concurrent peut s'approvisionner uniquement auprès de la RA et jouer sa stratégie optimale sans contrainte. Le régulateur a intérêt de fixer sa politique de régulation de manière à ce que le concurrent puisse acheter un maximum de quantités au prix $r^{s}$ pour maximiser les quantités vendues sur le marché aval et ainsi maximiser le bien-être.

Preuve. Annexe 2.

\subsection{L'équilibre du jeu : l'action du régulateur}

Le régulateur a pour objectif de maximiser le bien-être collectif en s'assurant que l'OH réalise des profits non négatifs. Une analyse des différents équilibres nous permet d'en écarter certains soit parce qu'ils sont d'un point de vue du régulateur et du welfare dominés, soit parce qu'ils ne sont pas impactés par la politique de RA.

D'abord, le welfare étant croissant en quantités, le régulateur sera incité à pratiquer une politique de régulation qui ne contraint pas le concurrent dans ses ventes. L'équilibre $\left(q_{e}^{s c *}, q_{o}^{s c *}\right)$ pour lequel les deux contraintes d'approvisionnement du concurrent sont saturées peut par conséquent être écarté car il est dominé par les autres équilibres.

Ensuite, lorsque $r^{s} \geq s$, le concurrent n'achète aucune quantité rétrocédée si $K$ est suffisamment élevé, c'est-à-dire lorsqu'il vérifie l'une des conditions $\frac{1}{2}-r^{s}<K<\frac{1}{2}-s$ ou $K \geq \frac{1}{2}-s$. Le régulateur ne pourra donc pas dans

\footnotetext{
${ }^{14}$ Nous retrouvons ici les équilibres présentés dans le cas pour lequel le concurrent ne peut s'approvisionner qu'auprès de la RA.
} 
ces cas financer les coûts échoués irrécupérables par une politique de $\mathrm{RA}^{15}$.

Enfin, lorsque $s \geq r^{s}$, le concurrent s'approvisionne en priorité auprès de la RA. Si $\alpha^{s} K_{o}$ est suffisamment élevé, c'est-à-dire $\frac{1}{2}-r^{s} \geq \alpha^{s} K_{o} \geq \frac{1}{2}-s$ ou $\alpha^{s} K_{o} \geq \frac{1}{2}-r^{s}$, le concurrent ne s'approvisionne pas auprès de la source alternative plus coûteuse. Les équilibres sont alors ceux du premier cas étudié (section 3).

Finalement, après cette analyse, les équilibres données par (Eq. 13) et (Eq.14) retiennent notre attention. Ce sont les équilibres pour lesquels le concurrent réalise un arbitrage et peut s'approvisionner auprès des deux sources d'approvisionnement.

Lorsque $r^{s} \geq s$, alors l'équilibre est $\left(q_{e 2}^{s *}, q_{o 2}^{s *}\right)$ (Eq.13). Le welfare est concave en $r^{s}$ et décroissant pour $r^{s} \in\left[\frac{1}{6}, \frac{1}{2}\right]$. Cet intervalle de variation découle de la positivité à la fois de $q_{e 2}^{s *}$ et de $\lambda_{e 2}^{s c *}$. Le régulateur doit donc adopter une politique qui minimise le prix de rétrocession $r^{s}$. Le prix de rétrocession d'équilibre va toujours se situer sur la partie décroissante du welfare. Ici, le seul prix acceptable dans l'intervalle est $r^{s *}=\frac{1}{6}$. Le concurrent joue alors sa stratégie $q_{e 2}^{s *}=\frac{1}{3}$ sur le marché aval. Pour ce prix, le profit de l'OH $\Pi_{o 2}^{s *}=\frac{1}{6}-u K_{o}-\frac{1}{6} K$. Il sera positif pour $u K_{o} \leq \frac{1}{6}-\frac{1}{6} K ;$ l'OH est suffisamment efficace pour que le profit de duopole suffise à financer les coûts échoués irrécupérables qui pourraient apparaître. Si le régulateur choisit un prix $r^{s} \in\left[0, \frac{1}{6}\left[\right.\right.$ avec $r^{s *} \geq s$, alors le concurrent peut jouer sa stratégie de Cournot sans contrainte sur le marché aval $q_{e 2}^{s *}=\frac{1}{3}\left(\operatorname{car} \frac{1}{2}-r^{s}>\frac{1}{3}\right)$. Etablir un prix $r^{s}$ dans cet intervalle n'a donc pas d'autres conséquences que de réaliser un transfert financier de l'OH vers le concurrent, les approvisionnements par la RA de ce dernier étant moins coûteux mais les stratégies sur le marché aval restant constantes.

En revanche, pour $u K_{o}>\frac{1}{6}-\frac{1}{6} K$, les coûts échoués irrécupérables font

\footnotetext{
${ }^{15}$ Comme évoqué dans la section 3 , nous n'analysons ici que le mécanisme de financement des coûts échoués irrécupérables par la RA. En effet, l'introduction d'un autre transfert entre les agents imposerait de modifier notre approche et notre jeu. Notamment, le concurrent anticipant la mise en place d'un transfert internaliserait cette nouvelle contrainte dans son programme d'optimisation, modifiant ses croyances et donc sa stratégie d'approvisionnement ou d'offre.
} 
subir à l'OH des pertes. Le financement de ces coûts échoués irrécupérables nécéssite une adaptation du prix de rétrocession. Le prix d'équilibre sera alors $r^{s *}=r_{2}^{s *}=\frac{1}{2}-\frac{2}{3} K-\frac{1}{3} \sqrt{3-6 K+4 K^{2}-12 u K_{o}}$, solution de l'équation $\Pi_{o 2}^{s *}=0$. La variable duale associée à ce prix $r_{2}^{s *}$ est $\gamma_{2}^{s *}=\frac{2 r_{2}^{s *}+1}{6-8 K-12 r_{2}^{s *}}>0$. Ce prix $r_{2}^{s *} \in\left[\frac{1}{6}, \frac{1}{2}\right]$ et, lorsqu'il existe, est toujours une solution pour $u K_{o}>$ $\frac{1}{6}-\frac{1}{6} K$.

Or, il n'est pas positif sur l'ensemble de l'intervalle de variation de $u K_{o} \in$ ]$\left.\frac{1}{6}-\frac{1}{6} K, \frac{1}{4}\right]$. En effet, ce prix sera solution lorsque $u K_{o} \in\left[\frac{1}{6}-\frac{1}{6} K, \frac{1}{4}-\frac{1}{2} K+\frac{1}{3} K^{2}\right]$ car il est positif. Lorsque $u K_{o} \in\left[\frac{1}{4}-\frac{1}{2} K+\frac{1}{3} K^{2}, \frac{1}{4}\right]$, ce prix $r_{2}^{s *}$ n'existe pas ; le régulateur ne peut alors pas financer les coûts échoués irrécupérables à l'aide de sa politique de régulation, l'OH étant trop inefficace.

Pour que $r_{2}^{s *}$ soit solution, il faut également qu'il permette de respecter la condition d'équilibre en sous-jeux $\frac{1}{2}-r^{s} \geq K$. Il existe un intervalle de variation de $u K_{o} \in\left[\frac{1}{4}(K-1)^{2} ; \frac{1}{4}-\frac{1}{2} K+\frac{1}{3} K^{2}\right]$ pour lequel le prix $r_{2}^{s *}$ existe mais ne permet pas au concurrent d'acheter des quantités car $K>\frac{1}{2}-r^{s}$. Comme nous l'avons vu, le concurrent préfère alors ne pas acheter de quantités rétrocédées $\left(K_{e}^{s *}=0\right)$ car ses achats auprès de la source alternative lui permettent d'acheter $K$ à un prix moins coûteux $s$. Ce niveau d'approvisionnement suffit pour qu'il puisse vendre davantage de quantités, indépendamment du prix de rétrocession déterminé dans l'intervalle $\left[\frac{1}{6}, \frac{1}{2}\right]$.

Finalement, trois situations d'équilibre résument ce cas (Fig. 1) :

- Si $\frac{1}{6}-\frac{1}{6} K \geq u K_{o}$, alors $r^{s *}=\frac{1}{6}$ et le profit de duopole finance les coûts échoués irrécupérables car l'OH est efficace. Tout prix $r^{s *}$ inférieur ne fait que modifier la répartition des profits entre l'OH et son concurrent, ce dernier pouvant jouer sa stratégie de meilleure réponse $q_{e}^{*}=\frac{1}{3}$.

- Si $\frac{1}{4}(K-1)^{2} \geq u K_{o}>\frac{1}{6}-\frac{1}{6} K$, alors $r_{2}^{s *} \geq \frac{1}{6}$ et le régulateur choisit $r_{2}^{s *}$ qui permet à l'OH de réaliser un profit nul.

- Si $u K_{o}>\frac{1}{4}(K-1)^{2}$, alors la contrainte d'existence de l'équilibre en sous-jeux $K \leq \frac{1}{2}-r^{s}$ n'est pas vérifiée. Le régulateur ne peut pas décider d'un prix de rétrocession appartenant à l'intervalle $\left[\frac{1}{6}, \frac{1}{2}\right]$ qui incite le concurrent à acheter des quantités rétrocédées. La source alterna- 
tive lui permet de vendre et d'atteindre des profits supérieurs à toutes situations dans laquelle il achèterait des quantités rétrocédées (pour augmenter ses ventes mais également en augmentant ses coûts). L'OH n'est pas efficace ce qui ne rend pas la rétrocession intéressante pour le concurrent. Le régulateur n'a pas d'incitation à décider une RA.

Pour maximiser le bien-être collectif, le régulateur définit une proportion minimale qui permet au concurrent de jouer ses stratégies de meilleures réponses sans contrainte, $q_{e 2}^{s *}=\frac{1}{2}-r^{s *}$. Par conséquent, $\alpha_{2}^{s *}$ doit être telle que $\alpha_{2}^{s *} K_{o}+K=\frac{1}{2}-r^{s} \Leftrightarrow \alpha_{2}^{s *}=\frac{1-2 K-2 r^{s *}}{2 K_{o}}$. Si le régulateur choisit $r^{s *}=\frac{1}{6}$, alors il détermine une proportion minimale $\alpha_{2}^{s *}=\frac{1-3 K}{3 K_{o}}$. Si le régulateur choisit $r^{s *}=r_{2}^{s *}$, alors la proportion rétrocédée est $\alpha^{s *}=\alpha_{2}^{s *}$ $=\frac{1}{3} \frac{\sqrt{3-6 K+4 K^{2}-12 u K_{o}}-K}{K_{o}}$. La rétrocession dans ces deux cas est efficace car elle conduit le concurrent à acheter les quantités rétrocédées et à réaliser un profit qui est toujours positif, à la fois pour $r^{s *}=\frac{1}{6}$ et $r^{s *}=r_{2}^{s *}$.

Conclusion 2 Dans la situation où le prix de rétrocession est supérieur à celui de la source alternative d'approvisionnement $\left(r^{s} \geq s\right)$, trois situations d'équilibre se distinguent. Lorsque $\frac{1}{6}-\frac{1}{6} K \geq u K_{o}$, alors le régulateur choisit $r^{s *}=\frac{1}{6}$ et laisse une rente à l'OH car il est efficace. Le régulateur maximise le welfare tout en permettant à l'OH de réaliser des profits positifs. Le régulateur choisit une proportion rétrocédée $\alpha_{2}^{s *}=\frac{1-3 K}{3 K_{o}}$. Lorsque $\frac{1}{4}(K-1)^{2} \geq u K_{o}>\frac{1}{6}-\frac{1}{6} K$, alors le régulateur fixe un prix $r^{s *}=r_{2}^{s *}$.Ce prix est celui de Ramsey-Boiteux, c'est-à-dire qu'il maximise le welfare en ne laissant aucune rente à l'OH. Il choisit une proportion $\alpha_{2}^{s *}$ telle que $\alpha_{2}^{s *}=\frac{1}{3} \frac{\sqrt{3-6 K+4 K^{2}-12 u K_{o}}-K}{K_{o}}$. Lorsque $u K_{o}>\frac{1}{4}(K-1)^{2}$, alors le régulateur n'a pas d'incitation à décider d'une $R A$ car le concurrent n'achète pas de quantités rétrocédées. 


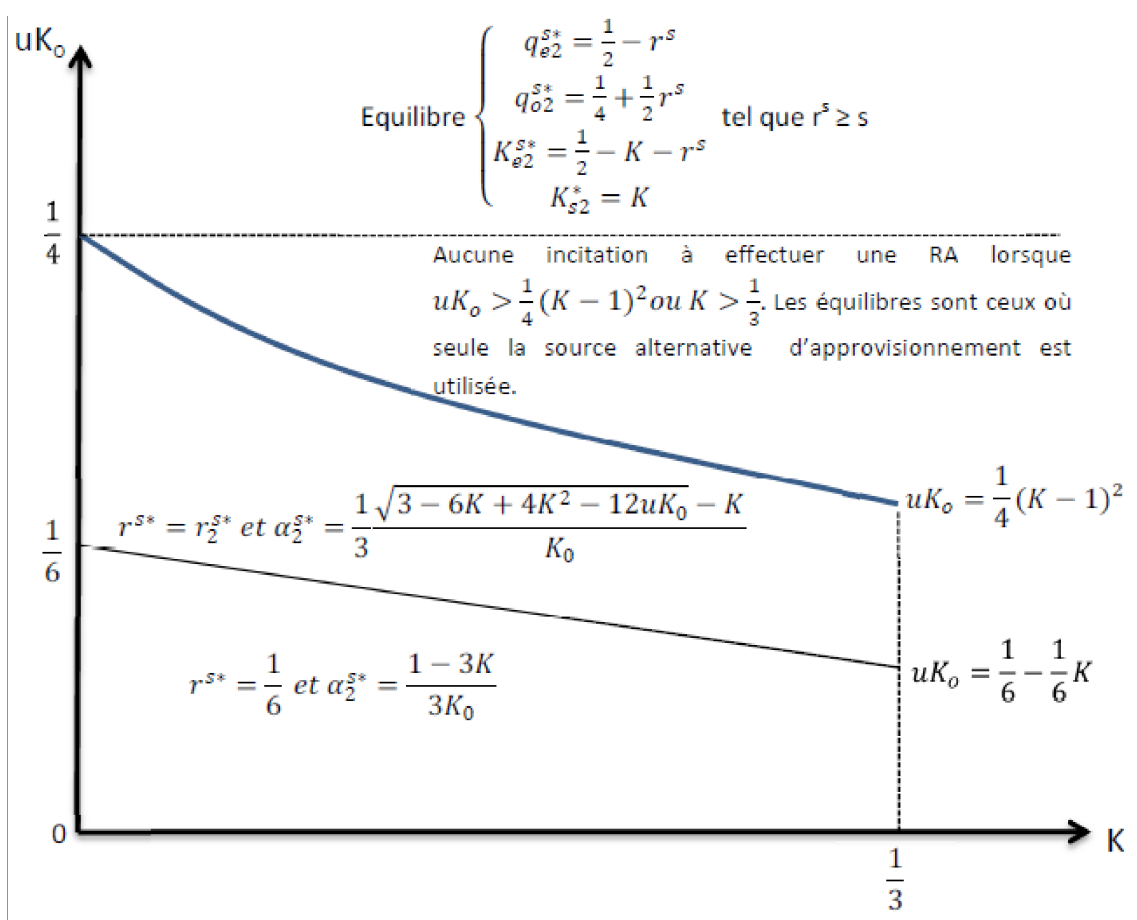

Fig. 1 : Equilibres avec approvisionnement alternatif $\left(r^{s} \geq s\right)$.

Si $s \geq r^{s}$, alors l'équilibre atteint est $\left(q_{e 3}^{s *}, q_{o 3}^{s *}\right)$ (Eq.14). Le concurrent achète toutes les quantités rétrocédées et le reste de ses ventes auprès de la source alternative. La condition de positivité sur les approvisionnements $K_{s 3}^{*}$ nous permet de déterminer une condition sur la proportion rétrocédée $\alpha_{3}^{s *}: K_{s} \geq 0 \Leftrightarrow \frac{1-2 s}{2 K_{o}} \geq \alpha_{3}^{s *}$. Le welfare étant croissant en $\alpha$, le régulateur sera incité à fixer la valeur maximale, c'est-à-dire $\alpha_{3}^{s *}=\frac{1-2 s}{2 K_{o}}$. En fixant cette proportion, le régulateur permet au concurrent de s'approvisionner à la source la moins coûteuse, c'est-à-dire auprès de la RA : $K_{s 3}^{*}=0$ et $q_{e}^{s *}=\alpha_{3}^{s *} K_{o}$. En prenant cette décision, il optimise l'efficacité de l'activité.

Le bien-être collectif ne dépend pas ici du prix de rétrocession. Le régulateur peut donc fixer un prix $r^{s *}$ ne respectant que la condition d'être inférieur à $s$. Cependant, comme dans les équilibres précédents, l'OH est susceptible d'encourir des coûts échoués irrécupérables que le régulateur est incité à financer pour éviter la monopolisation de l'activité. Il peut donc choisir un 
prix $r^{s *}=r_{3}^{s *}=\frac{-4 s+16 u K_{o}-4 s^{2}-1}{16-32 s}$ solution de l'équation $\Pi_{o 3}^{s *}\left(q_{e 3}^{s *}, q_{o 3}^{s *}\right)=0$. Nous allons calculer deux valeurs seuil ${ }^{16}$. La première, $s_{1}=2 \sqrt{u K_{o}}-\frac{1}{2}$, est telle que si $s \leq s_{1}$, alors $r_{3}^{s *} \geq 0$. La seconde, $s_{4}=\frac{5}{14}-\frac{2}{7} \sqrt{2-7 u K_{o}}$, est telle que si $s<s_{4}$, alors $r_{3}^{s *}>s$. La discussion comprend alors plusieurs cas énoncés ci-après pour le choix du prix de rétrocession (Fig. 2) :

- Si $s \leq \frac{1}{6}$, l'équilibre $\left(q_{e 3}^{s *}, q_{o 3}^{s *}\right)$ n'est pas atteignable. Ce cas renvoie à ceux étudiés en l'absence de source alternative d'approvisionnement (section 3).

- Si $s \in\left[\frac{1}{6}, s_{4}\left[, r_{3}^{s *}>s\right.\right.$ donc le régulateur ne peut pas choisir le prix qui permet de financer les coûts échoués irrécupérables. L'OH est trop inefficace pour qu'il puisse réaliser des profits positifs, sachant que les prix sur le marché alternatif ne sont pas très élevés. Il peut établir un prix $r^{s}<s$ mais l'OH réalise des pertes. $r_{3}^{s *}>s$ nous renvoie au cas précédent.

- Si $s \in\left[s_{4}, s_{1}\right], 0 \leq r_{3}^{s *} \leq s$ donc le régulateur peut financer les coûts échoués irrécupérables de l'OH sachant que les prix de la source alternative sont plus élevés. Le prix d'équilibre est donc $r^{s *}=r_{3}^{s *}$.

- Si $\left.s \in] s_{1}, \frac{1}{2}\right], r_{3}^{s *}<0$ donc le régulateur choisit un prix $r^{s *}=0$ ou $r^{s *}=u$. Le profit de l'OH est positif car les prix de la source alternative sont très élevés.

L'analyse de ce cas nous montre que le prix de la source alternative joue ici un rôle essentiel aux côtés des coûts de l'OH. En effet, pour des niveaux de prix $s$ élevés ou de coûts de l'OH faibles, le régulateur pourra toujours financer les coûts échoués irrécupérables par sa politique de régulation. Un prix élevé de la source alternative agit comme un coût d'opportunité pour le concurrent. Sa disposition à payer pour son approvisionnement auprès de la RA augmente. Son profit étant toujours positif, il peut réaliser son activité et dispose donc d'une incitation à acheter les quantités rétrocédées pour être

\footnotetext{
${ }^{16}$ Ces valeurs seuils et les bornes définissant les intervalles de variation de $u K_{o}$ et de $s$ sont calculées de manières à ce que les prix de rétrocession définis existent et respectent les différentes conditions d'équilibre du marché des approvisionnements (annexe 3).
} 
actif sur le marché. La seule situation pour laquelle le régulateur ne peut pas financer ces coûts échoués irrécupérables émerge lorsque les coûts $u K_{o}$ sont élevés et le prix de la source alternative d'approvisionnement faible. Cette analyse est intuitive. En effet, la disposition à payer du concurrent pour les quantités rétrocédées est plus faible étant donné des coûts d'accès à la source alternative peu élevés. De plus, le prix $r_{3}^{s *}>s$ dans cette situation ce qui nous renvoie aux équilibres du cas précédent. Le régulateur peut fixer des prix $r^{s}<r_{3}^{s *}$ mais ne finance pas les coûts échoués irrécupérables.

Nous avons vu que $r_{3}^{s *}$ n'était pas toujours atteignable. Certaines situations permettent à l'OH de réaliser des profits positifs, notamment lorsque le prix sur le marché alternatif est très élevé et ses conditions d'approvisionnement efficaces. Le régulateur peut pratiquer $r^{s *}=u$ ou égal au coût marginal d'approvisionnement, qui est ici nul. Fixer un prix de rétrocession plus élevé n'aura pour incidence que de créer des transferts de profit de l'OH vers le concurrent. Le profit de l'OH étant croissant en $r^{s}$, ce profit est toujours positif sauf lorsque $r^{s}=r_{3}^{s *}$, prix pour lequel il est nul, ou lorsque $\frac{1}{4} \geq u K_{o}>\frac{2}{9}$ et $s \in\left[\frac{1}{6}, s_{4}\right.$ [, pour lequel il est négatif. Les intervalles de positivité élevés proviennent du fait que les stratégies des acteurs dépendent du prix d'achat des quantités additionnelles auprès de la source alternative d'approvisionnement. Dès lors, et comme $K_{o}$ ne contraint jamais les acteurs, le régulateur adopte une RA bénéfique à la fois pour le concurrent (il achète ses quantités d'équilibre à un prix de rétrocession moins cher que $s$ ), pour l'OH (il vend une partie de ses approvisionnements d'autant plus cher que $s$ est élevé et donc dispose d'une rente plus élevée) et pour le bien-être collectif (les coûts des approvisionnements des acteurs sont minimisés et les ventes maximales). Nous avons vu que la proportion optimale rétrocédée était $\alpha^{s *}=\frac{1-2 s}{2 K_{o}}$. Fixer une proportion plus élevée n'aura que pour incidence de laisser certaines quantités rétrocédées non achetées.

Conclusion 3 Le régulateur peut financer les coûts échoués irrécupérables lorsque l'OH est efficace ou lorsque le prix s de la source alternative est 
élevé. Lorsque l'OH est très inefficace ou le prix de la source alternative faible, alors il lui est impossible de calculer un prix de rétrocession finançant ces coûts échoués irrécupérables.

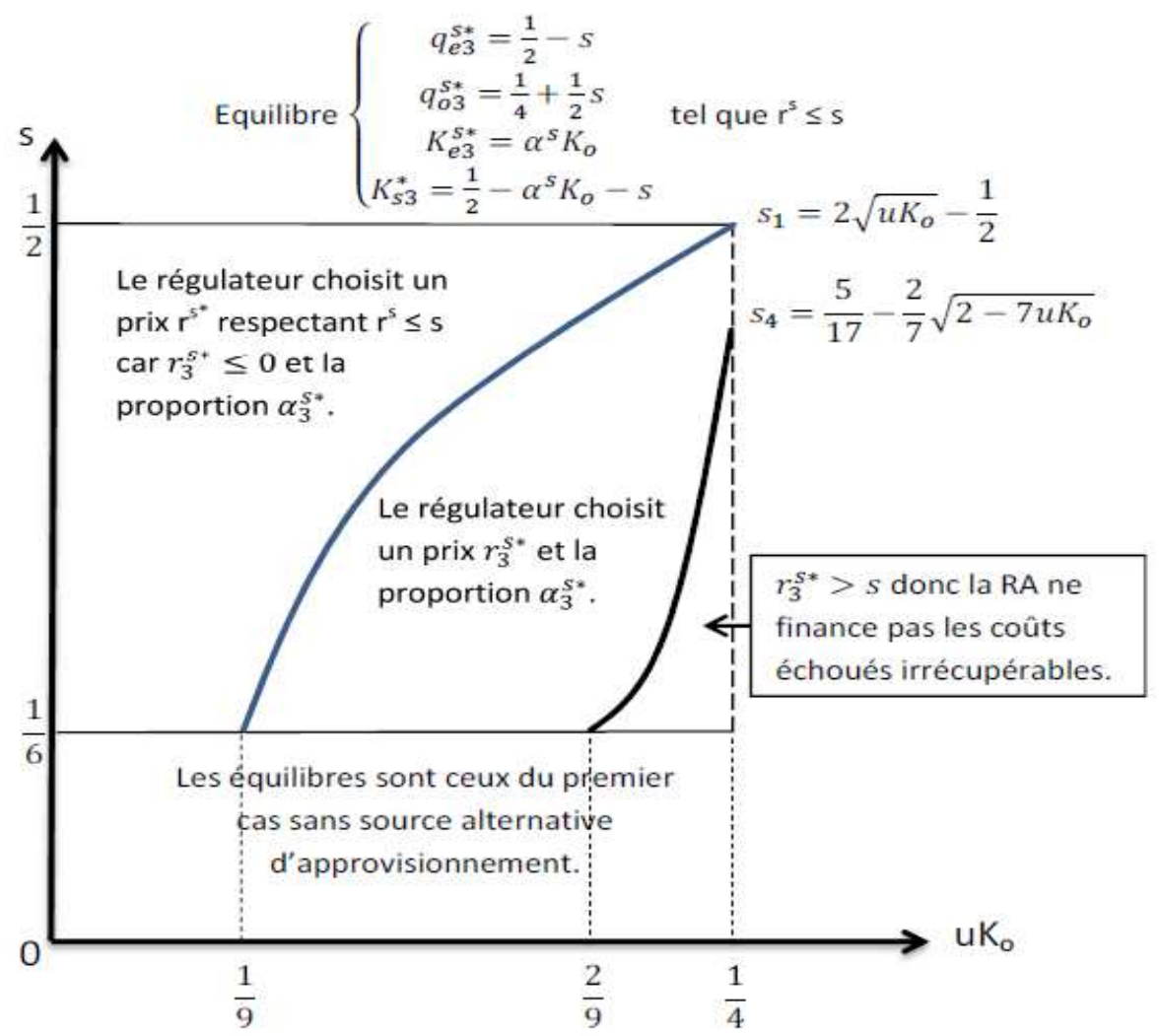

Fig. 2 : Equilibres avec approvisionnement alternatif $\left(r^{s} \leq s\right)$.

Proposition 2 Le régulateur tient compte des sources alternatives d'approvisionnement pour établir sa politique de régulation. Lorsque $s \geq r^{s *}$, alors le régulateur cherche à minimiser le coût de l'activité. Lorsque le prix s est abordable, le régulateur ne peut pas financer les coûts échoués irrécupérables de l'OH si ce dernier est inefficace. 


\section{Preuve. Annexe 3.}

L'analyse de la répartition des surplus entre les agents lorsque $r^{\mathbf{*}} \leq s$ est la même que celle du cas précédent (section 3). En effet, le régulateur n'a aucune incitation à contraindre le concurrent dans ses achats de quantités à l'OH. Il fixe donc une politique de régulation asymétrique qui permet à chaque opérateur d'offrir sa stratégie de meilleure réponse sur le marché. Lorsque $r^{s} \geq s$, la régulation asymétrique a des bienfaits sur le bien-être uniquement lorsque le concurrent est contraint. Ce dernier trouve alors une possibilité d'accroître son profit, les consommateurs étant également davantage satisfaits par l'augmentation des ventes sur le marché final. L'OH trouve également positif cette rétrocession qui peut lui occasionner des rentes, rentes corrélées positivement avec l'efficacité de ses approvisionnements.

\section{Conclusion}

L'application d'une RA permet de rendre les concurrents actifs sur le marché. L'impact sur la concurrence est positif. Cependant, il est nécessaire pour le régulateur de contrôler ces entrées pour éviter deux types d'écueils. Le premier est que l'entrant ne s'avère en réalité inefficace. Les efficacités productive et allocative sont alors impactées négativement par cette entrée. La seconde est d'éviter des comportements collusifs, la RA créant des interactions supplémentaires entre les agents. Ces stratégies sont d'autant plus probables lorsque ce sont les capacités de production de l'OH qui sont rétrocédées. La mise en place de cette concurrence forcée modifie artificiellement le contexte concurrentiel et réglementaire dans lequel les acteurs opèrent. Certains investissements effectués par l'OH sont susceptibles de se trouver non rentables. Ces coûts échoués irrécupérables pénalisent l'opérateur en place. Nous avons montré que le régulateur peut alors établir une politique de régulation optimale qui permette au concurrent d'être actif sur le marché de détail tout en finançant ces coûts échoués irrécupérables. Les résultats 
montrent une relation croissante entre efficacité de l'OH et de la RA. Si les coûts d'approvisionnement de l'OH sont efficaces, alors le régulateur peut systématiquement maximiser le welfare et éviter le poids de ces coûts échoués irrécupérables en les finançant à l'aide du prix de rétrocession. En cas de forte efficacité, l'OH conserve même une rente, les profits de duopole étant suffisant pour rentabiliser l'activité de l'OH. En cas de moindre efficacité, les solutions trouvées seront celles de Ramsey-Boîteux. En effet, le régulateur aura alors à fixer un prix de rétrocession régulé du type Ramsey-Boîteux pour maximiser le welfare et conserver des profits au moins nuls pour l'OH. En présence d'une source alternative d'approvisionnement à la régulation asymétrique, la politique de régulation dépend du prix de cette nouvelle source disponible. Lorsque le prix de la source alternative est inférieur au prix de rétrocession, des prix Ramsey-Boîteux permettent de financer les coûts échoués irrécupérables. L'offre du concurrent se répartit alors entre achat de la quantité maximale sur le marché alternatif et achat du complément par l'intermédiaire de la RA. Lorsque le prix de la source alternative d'approvisionnement est plus élevé que le prix de rétrocession, le régulateur, pour conserver l'efficacité de la mesure et de l'activité, fixe sa politique de manière à ce que le concurrent puisse s'approvisionner uniquement auprès de l'OH à un faible coût.

\section{Références}

Armstrong M., Cowan S., Vickers J. [1994], Regulatory Reform : Economic Analysis and British Experience, Cambridge, MIT Press.

Armstrong M. [1999], Regulation and Inefficient Entry : Economic Analysis and British Experience, Oxford, Nuffield College.

Baumol W. J., Sidak J. G. [1995], "Stranded Costs", Harvard Journal of Law \& Public Policy,18(3), p. 835-849.

Beard T.R., Kaserman D.L., Mayo J.W. [2003], "Regulation, competition, and the optimal recovery of stranded costs", International Journal of 
Industrial Organization, 21(10), p. 831-848.

BeEsLey M.E. [1997], Privatization, regulation and deregulation, second ed., London, Routledge.

Bernheim B.D., Whinston M.D. [1990], "Multimarket contact and collusive behaviour", Rand Journal of Economics, 21(1), p. 1-26.

BerRY S.K. [2000], "Stranded costs, access charges, and ramsey pricing in the U.S. electric utility industry", Quarterly Review of Economics and Finance, 40(4), p. 503-517.

Bremberger C., Bremberger F., Rammerstorfer M. [2012], "The Impact of Different Unbundling Scenarios on Wholesale Prices in Energy Markets", Energy Journal, 33(3), p. 183-214.

Breton M., Zaccour G. [2001], "Equilibria in an asymmetric duopoly facing a security constraint", Energy Economics, 23(4), p. 457-475.

Brock W.A., Scheinkman J.A. [1985], "Price Setting Supergames with Capacity Constraints", Review of Economic Studies, 52(3), p. 371-382.

Carsberg B. [1993], "Promoting entry into regulated industries", in M. Beesley (ed.), Majors Issues in Regulation, London, Institute of Economic Affairs, p. 89-98.

Chaton C., Gasmi F., Gulllerminet M-L., Oviedo J-D. [2008], "Un instrument de court terme pour stimuler la concurrence : le gas release", Revue économique, 59(3), p. 475-486.

Chaton C., Gasmi F., Guillerminet M-L., Oviedo J-D. [2012], "Gas release and transport capacity investment as instruments to foster competition in gas market", Energy Economics, 34(5), p. 1251-1258.

Clastres C. [2005], Le gas release comme facteur d'incitation à la concurrence dans l'industrie gazière européenne, $\mathrm{PhD}$ Thesis, Montpellier, Université I.

Clastres C., David L. [2009], "The impact of asymmetric regulation on surplus and welfare : the case of gas release programmes", Opec Energy Review, 33(2), p. 97-110.

Compte O., Jenny F., Rey P. [2002], "Capacity constraints, mergers 
and collusion", European Economic Review, 46(1), p. 1-29.

Cramton P., Stoft S. [2005], "A Capacity Market that Makes Sense", The Electricity Journal, 18(7), p. 43-54.

Creti A., Pouyet J., Sanin M-E. [2013], "The NOME law : implications for the French electricity market", Journal of regulatory Economics, 43(2), April 2013, p. 196-213.

Davidson C., Deneckere R.J. [1986], "Long-run competition in capacity, short-run competition in price, and the Cournot model", Rand Journal of Economics, 17(3), p. 404-415.

European Commission [2007], "DG Competition Report on Energy Sector Enquiry", DG Competition, Brussels, January 10.

Green R., Newberry D. [1992], "Competition in the British Electricity spot Market", Journal of Political Economy, 100(5), p. 929-953.

Ivaldi M., Jullien B., Rey P., Seabright P., Tirole J. [2003a], "The Economics of Tacit Collusion", Final Report for DG Competition, European Commission, March.

Ivaldi M., Jullien B., Rey P., Seabright P., Tirole J. [2003b], "The Economics of Unilateral Effects", Final Report for DG Competition, European Commission, November.

Joskow P.L. [1996], "Does Stranded Cost Recovery Distort Competition?", Electricity Journal, 9(3), p. 31-45.

Joskow P.L. [2008a], "Incentive Regulation and Its Application to Electricity Networks", Review of Networks Economics, 7(4), p. 547-560.

Joskow P.L. [2008b], "Lessons Learned From Electricity Market Liberalization", The Energy Journal, Special

Issue : The Future of Electricity : Papers in Honor of David Newberry, p. $9-42$.

Kolbe A.L., Tye W.B. [1996], "Compensation for the risk of stranded costs", Energy Policy, 24(12), p. 1025-1050.

Lesser J., Ainspan M. [1996], "Using Markets to Value Stranded Costs", Electricity Journal, 9(8), p. 66-74. 
Levitan R., Shubik M. [1972], "Price duopoly and capacity constraints", International Economic Review, 13(1), p. 111-122.

Lyon T. P., Huang H. [1995], "Asymmetric regulation and incentives for innovation", Industrial and corporate change, 4(4), p. 769-776.

Maloney M.T., Sauer, R. D. [1998], "A Principal Approach to the Stranded Cost Issue", Electricity Journal, 11(3), p. 58-64.

Penard T. [1997], "Choix de capacités et comportements stratégiques. Une approche par les jeux répétés", Annales d'économie et de statistique, 46, p. 203-224.

Penard T., Souam S. [2002], "Collusion et politique de la concurrence en information asymétrique", Annales d'économie et de statistique, 66, p. 209-233.

Percebois J. [2001], "Energie et théorie économique : un survol", Revue d'Economie Politique, 2001/6, 111, p. 815-860.

Perrucci A., Cimatoribus M. [1997], "Competition, convergence and asymmetry in telecommunications regulation", Telecommunications Policy, 21(6), p. 493-512.

SCHANKerman M. [1996], "Symetric regulation for competitive telecommunications", Information Economics and Policy, 8(1), p. 3-23.

Schankerman M., Waverman L. [1997], "Asymmetric Regulation, Asymmetric Information and Competition in Multimedia Markets". http ://businessinnovation.berkeley.edu/crtp/publications/asymreg.pdf

SmeErs Y., 1997, "Computable equilibrium models and the restructuring of the european electricity and gas market", Energy journal, 18(4), p. 1-31.

Tye W.B., Graves F.C. [1996], "Stranded Cost Recovery and Competition on Equal Terms", Electricity Journal, 9(10), p. 61-70. 


\section{Annexes}

\subsection{Annexe 1 : les prix Ramsey-Boîteux}

Le programme d'optimisation s'écrit :

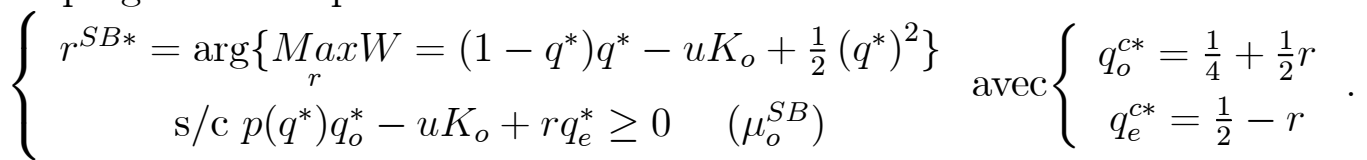

Les conditions nécessaires de premier ordre se déduisent :

$\left\{\begin{array}{l}\frac{d L_{o}}{d r}=\frac{3}{4} \mu_{o}-\frac{1}{4} r-\frac{3}{2} r \mu_{o}-\frac{1}{8}=0 \\ \mu_{o}^{S B}\left[p\left(q^{*}\right) q_{o}^{*}-u K_{o}+r q_{e}^{*}\right] \geq 0\end{array}\right.$

La contrainte libre nous renvoie au cas d'un profit pour l'OH $\Pi_{o}^{*}=\frac{1}{6}-$ $u K_{o}$, positif pour $u K_{o} \in\left[0, \frac{1}{6}\right]$.

La contrainte saturée implique que $\mu_{o}^{S B}>0$. Le profit de l'OH se doit alors d'être nul : $\Pi_{o}^{S B}=-\frac{3}{4} r^{2}+\frac{3}{4} r-u K_{o}+\frac{1}{16}=0$. Cette équation nous donne deux solutions $r_{1}^{S B}=\frac{1}{2}+\frac{1}{3} \sqrt{3-12 u K_{o}}$ et $r_{2}^{S B}=\frac{1}{2}-\frac{1}{3} \sqrt{3-12 u K_{o}}$.

Le seul prix qui soit possible est $r^{S B *}=r_{2}^{S B}=\frac{1}{2}-\frac{1}{3} \sqrt{3-12 u K_{o}}$ avec $u K_{o}>\frac{1}{6}$ car $r_{1}^{*}>\frac{1}{2}$. Cette valeur nous permet de calculer la variable duale $\mu_{o}^{S B *}=\frac{1}{6} \frac{\sqrt{3}-\sqrt{1-4 u K_{o}}}{\sqrt{1-4 u K_{o}}}$ positive pour $u K_{o}<\frac{1}{4}$.

\subsection{Annexe 2 : choix des stratégies d'approvisionne- ment avec une alternative d'approvisionnement}

Les équilibres en sous-jeu sur le marché final sont $\left\{\begin{array}{l}q_{e}^{s *}=\frac{1}{3} \\ q_{o}^{s *}=\frac{1}{3}\end{array}\right.$ si $\left(K_{e}^{s}+\right.$ $\left.K_{s}\right)>\frac{1}{3}$ et $\left\{\begin{array}{c}q_{e}^{s c}=K_{e}^{s}+K_{s} \\ q_{o}^{s c}=\frac{1}{2}-\frac{1}{2}\left(K_{e}^{s}+K_{s}\right) \quad \text { si }\left(K_{e}^{s}+K_{s}\right) \leq \frac{1}{3} . \\ \lambda_{e}^{s c}=-\frac{3}{2}\left(K_{e}^{s}+K_{s}\right)+\frac{1}{2}\end{array}\right.$

Le concurrent choisit son niveau d'approvisionnement en maximisant son profit par rapport à $K_{e}^{s}$ et $K_{s}$. Son programme est :

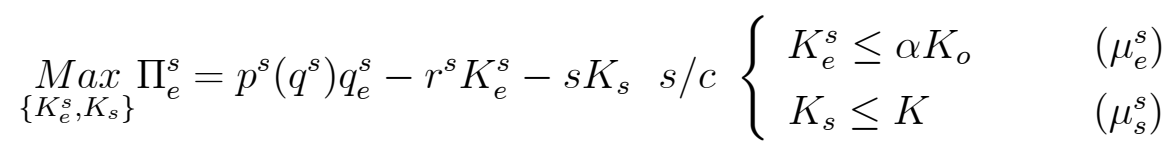


L'équilibre $\left(q_{o}^{s *}, q_{e}^{s *}\right)$ est impossible, les variables duales étant négatives. Dans

le cas de l'équilibre $\left(q_{o}^{s c *}, q_{e}^{s c *}\right)$, le lagrangien sera $L_{e}^{s}=\left(\frac{1}{2}-\frac{1}{2}\left(K_{e}^{s}+K_{s}\right)\right)\left(K_{e}^{s}+\right.$ $\left.K_{s}\right)-r^{s} K_{e}^{s}-s K_{s}+\mu_{e}^{s}\left(\alpha^{s} K_{o}-K_{e}^{s}\right)+\mu_{s}^{s}\left(K-K_{s}\right)$.

Les conditions de premier ordre sont :

$$
\left\{\begin{array}{c}
\frac{d L_{e}^{s}}{d K_{e}^{s}}=\frac{1}{2}-K_{e}^{s}-K_{s}-r^{s}-\mu_{e}^{s}=0 \\
\frac{d L_{e}^{s}}{d K_{s}}=\frac{1}{2}-K_{e}^{s}-K_{s}-s-\mu_{s}^{s}=0 \\
\mu_{e}^{s}\left(\alpha^{s} K_{o}-K_{e}^{s}\right)=0 \\
\mu_{s}^{s}\left(K-K_{s}\right)=0
\end{array} .\right.
$$

Lorsque la contrainte de RA et celles de la source alternative sont libres, une solution existe si et seulement si $r^{s}=s$. Si $r^{s}=s$, alors le concurrent achète indifféremment auprès des deux sources une quantité globale $K_{e}^{s}+$ $K_{s}=\frac{1}{2}-r^{s}$. Il n'achète pas toutes les quantités mises à sa disposition entre les deux sources. Les quantités totales vendues sont $q^{s *}=\frac{3}{4}-\frac{1}{2} r^{s}$.

Lorsque seule la contrainte de l'approvisionnement alternatif est saturée, alors les conditions de premier ordre nous permettent de calculer $K_{e}^{s}=\frac{1}{2}-$ $K-r^{s}$ et $\mu_{s}^{s *}=-s+r^{s}>0$. L'équilibre est $\left(q_{e 2}^{s *}, q_{o 2}^{s *}\right)=\left(\frac{1}{2}-r^{s}, \frac{1}{4}+\frac{1}{2} r^{s}\right)$. Il existe pour $\lambda_{e}^{s c}>0$ et $q_{e 2}^{s *}>0$, c'est-à-dire pour $r^{s} \in\left[\frac{1}{6}, \frac{1}{2}[\right.$.

Cet équilibre impose que $K_{e}^{s}=\frac{1}{2}-K-r^{s} \geq 0 \Leftrightarrow \frac{1}{2}-r^{s} \geq K$ et que $r^{s} \geq s$. Si $K>\frac{1}{2}-r^{s}$ ( $K$ est suffisamment grand) et que $r^{s} \geq s$, alors le concurrent achète tous ses approvisionnements auprès de la source alternative et ne va rien acheter auprès de la régulation asymétrique trop coûteuse. Dès lors, le régulateur n'a aucune incitation à adopter une politique de RA qui perd son sens.

Pour résumer, lorsque $r^{s} \geq s$ :

- Si $K \leq \frac{1}{2}-r^{s}$, le concurrent achète $K_{e}^{s}=\frac{1}{2}-K-r^{s}$. La vente d'une unité supplémentaire compense son coût d'achat $r^{s}$ car les volumes disponibles sur le marché alternatif sont faibles ;

- Si $\frac{1}{2}-s>K \geq \frac{1}{2}-r^{s}$, le concurrent achète $K_{e}^{s}=0$ et $K_{s}=K$. La RA ne sert pas à approvisionner le concurrent qui préfère acheter les quantités $K$ auprès de la source alternative. La question de la pertinence de la 
RA se pose. En effet, le concurrent est contraint car il ne peut acheter les volumes correspondant à sa stratégie de meilleure réponse. Le prix de rétrocession est trop élevé pour qu'il intègre à ses achats une part de quantités rétrocédées.

- Si $K \geq \frac{1}{2}-s$, le concurrent achète $K_{e}^{s}=0$ et $K_{s}=\frac{1}{2}-s$ ce qui lui permet de jouer sa stratégie de meilleure réponse sur le marché aval sans subir de contrainte d'approvisionnement. La quantité $K_{s}=$ $\frac{1}{2}-s$ est l'équilibre trouvé lorsque le concurrent n'a accès qu'à la source alternative d'approvisionnement. La RA est ici inutile car le concurrent peut vendre sur le marché aval sa stratégie optimale en achetant toutes ses quantités auprès de la source alternative la moins chère.

Lorsque seule la contrainte d'approvisionnement par la RA est saturée, alors les conditions de premier ordre nous permettent de calculer un nouvel équilibre tel que $K_{s}=\frac{1}{2}-\alpha^{s} K_{o}-s$ et $\mu_{e}^{s *}=s-r^{s}>0$. L'équilibre est $\left(q_{e 3}^{s *}, q_{o 3}^{s *}\right)=\left(\frac{1}{2}-s, \frac{1}{4}+\frac{1}{2} s\right)$. Il existe pour $\lambda_{e}^{s c}>0$ et $q_{e 3}^{s *}>0$, c'est-à-dire pour $s \in\left[\frac{1}{6}, \frac{1}{2}[\right.$.

Cet équilibre existe si $K_{s}=\frac{1}{2}-\alpha^{s} K_{o}-s \geq 0 \Leftrightarrow \frac{1}{2}-s \geq \alpha^{s} K_{o}$ avec $s \geq r^{s}$. Si $\alpha^{s} K_{o}>\frac{1}{2}-s$ avec $s \geq r^{s}$, alors le concurrent achète la totalité des quantités $\frac{1}{2}-s$ qu'il revend sur le marché aval auprès de la RA, le prix d'achat étant plus faible que celui de la source alternative. Dès lors, les équilibres sont ceux du cas sans source alternative d'approvisionnement (section 3 et annexe 1) car il souhaiterait pouvoir acheter $\frac{1}{2}-r^{s}$ qui est supérieur à $\frac{1}{2}-s$. Dès lors, lorsque $\frac{1}{2}-r^{s}>\alpha^{s} K_{o}>\frac{1}{2}-s$, le concurrent achète la totalité des quantités rétrocédées pour vendre sur le marché final $q_{e 3}^{s *}=q_{e}^{c *}=\alpha^{s} K_{o}$. Lorsque $\alpha^{s} K_{o} \geq \frac{1}{2}-r^{s}$, alors le concurrent achète $K_{e 3}^{s *}=K_{e}^{*}=\frac{1}{2}-r^{s}$ et vend sur la marché final $q_{e 3}^{s *}=q_{e}^{c *}=\frac{1}{2}-r^{s}$.

Lorsque ces deux contraintes sont saturées, les conditions de premier ordre déterminent les multiplicateurs associés qui sont $\left\{\begin{array}{l}\mu_{e}^{s *}=\frac{1}{2}-\alpha^{s} K_{o}-K-r^{s} \\ \mu_{s}^{s *}=\frac{1}{2}-\alpha^{s} K_{o}-K-s\end{array}\right.$. Le concurrent achète toutes les quantités mises à sa disposition. Les deux multiplicateurs doivent être positifs, ce qui nous donne les deux conditions 
suivantes à respecter $\left\{\begin{array}{l}\alpha^{s} K_{o}+K<\frac{1}{2}-r^{s} \\ \alpha^{s} K_{o}+K<\frac{1}{2}-s\end{array}\right.$. Ces conditions s'interprètent facilement. Lorsque les approvisionnements disponibles pour le concurrent sont inférieurs à la quantité qu'il aurait désiré jouer soit lorsque le prix pratiqué sur le marché spot est inférieur à celui de la rétrocession (c'est-à-dire à la quantité $q^{s *}=\frac{1}{2}-s$ ), soit lorsque le prix de rétrocession est inférieur à celui du spot (c'est-à-dire à la quantité $q^{s *}=\frac{1}{2}-r^{s}$ ), alors le concurrent achète toutes les quantités qui lui sont disponibles. La quantité totale mise sur le marché est alors $q^{s *}=\frac{1}{2}+\frac{1}{2}\left(\alpha^{s} K_{o}+K\right)$.

\subsection{Annexe 3 : régulation optimale avec une alterna- tive d'approvisionnement}

Comme nous l'avons indiqué au sein de l'article, nous allons ici nous concentrer sur les cas de l'annexe 2 pour lesquels $K \leq \frac{1}{2}-r^{s}$ et $\frac{1}{2}-s \geq \alpha^{s} K_{o}$. En effet, les autres cas peuvent être écartés de l'analyse car ils sont soit dominés, soit renvoie au cas développé dans la section 3 , soit conduisent à la conclusion que la mise en place de la RA est inutile. Dès lors, le financement des coûts échoués irrécupérables par cette politique de régulation n'est pas envisagé.

Le régulateur maximise le welfare sous contrainte de profit positif pour l'OH.

$$
\underset{\left\{\alpha^{s}, r^{s}\right\}}{\operatorname{Max}} W^{s} \mathrm{~s} / \mathrm{c} \Pi_{o}^{s} \geq 0\left(\gamma^{s}\right) \text { avec }\left\{\begin{array}{c}
W^{s}=\Pi_{o}^{s}+\Pi_{e}^{s}+S_{c}^{s} \\
\Pi_{o}^{s}=p^{s}\left(q^{s}\right) q_{o}^{s}+r^{s} K_{e}^{s}-u K_{o}
\end{array}\right.
$$

Le welfare est égal à $W^{s}=\left(1-q^{s}\right) q^{s}-u K_{o}-s K_{s}+\frac{1}{2}\left(q^{s}\right)^{2}$.

$\frac{d W^{s}}{d q^{s}}=1-q^{s}>0$, le welfare est croissant en quantités pour notre étude. Les conditions d'approvisionnements $u K_{o} \in\left[0, \frac{1}{4}\right]$.

$$
\underset{\left\{\alpha^{s}, r^{s}\right\}}{\operatorname{Max}} W^{s} \Rightarrow\left\{\begin{array}{l}
\frac{d W^{s}}{d \alpha^{s}}=0 \\
\frac{d W^{s}}{d r^{s}}=0
\end{array} \text { s/c } \Pi_{o}^{s} \geq 0\left(\gamma^{s}\right)\right.
$$

L'expression du welfare lorsque les deux contraintes sont saturées est $W_{1}^{s}=p\left(q^{s}\right) q^{s}-u K_{o}^{s}-s K_{s}+\frac{1}{2}\left(q^{s}\right)^{2}$. Pour maximiser ce welfare, le régulateur 
n'a pas intérêt à réduire ou contraindre les ventes sur le marché final car $\frac{d W^{s}}{d q^{s}}>0$. Il n'a aucune incitation à adopter une RA $\left(\alpha^{s}, r^{s}\right)$ qui soit telle qu'elle contraindra le concurrent si ce dernier n'a pas accès à une autre source compétitive et en quantité suffisante.

\section{La contrainte $K_{s}=K$ est seule saturée}

L'équilibre en sous-jeu est $\left(q_{e 2}^{s *}, q_{o 2}^{s *}\right)$. Lorsque la contrainte de la source alternative est saturée, $W_{2}^{s}=\frac{15}{32}-\frac{1}{8} r^{s}-\frac{1}{8}\left(r^{s}\right)^{2}-u K_{o}-s K$.

$\frac{d W_{2}^{s}}{d r^{s}}=-\frac{1}{8}-\frac{1}{4} r^{s}=0 \Leftrightarrow r^{s}=-\frac{1}{2}$ ce qui est impossible donc pour $r^{s}>0$ le welfare est décroissant en $r^{s}$. Le régulateur souhaiterait choisir $r^{s *}=0$, c'est-à-dire un prix égal au coût marginal de l'OH. Or, l'équilibre en sousjeu n'existe que pour $r^{s *} \geq \frac{1}{6}$. Le régulateur choisira donc $r^{s *}=\frac{1}{6}$. Choisit $r^{s *}=0$ ne permet pas d'atteindre l'équilibre. En effet, l'inégalité $s>r^{s *}$ sera alors vérifiée.

La fonction de profit de l'OH s'écrit $\Pi_{o}^{s}=\left(1-q^{s}\right)\left(q_{o}^{s}\right)-u K_{o}+r^{s}\left(K_{e}^{s}\right) \Leftrightarrow$ $\Pi_{o}^{s}=\frac{1}{16}+\frac{3}{4} r^{s}-\frac{3}{4} r^{s} 2-u K_{o}-r^{s} K$. Pour $r^{s *}=\frac{1}{6}, \Pi_{o}^{s *}=\frac{1}{6}-u K_{o}-\frac{1}{6} K$. Il sera positif pour $u K_{o} \leq \frac{1}{6}-\frac{1}{6} K$. Pour $u K_{o}>\frac{1}{6}-\frac{1}{6} K$, le régulateur pourra mettre en place un système de tarification Ramsey-Boîteux pour financer les coûts échoués irrécupérables de l'OH. Toutefois, le welfare étant décroissant, il choisira toujours le plus petit prix de rétrocession possible pour le maximiser.

Cette fonction $\Pi_{o}^{s}$ admet comme maximum $r^{s \max }=\frac{1}{2}-\frac{2}{3} K$. Résoudre $\Pi_{o}^{s}=0$ conduit à deux racines solutions :

$$
\left\{\begin{array}{l}
r_{1}^{s *}=\frac{1}{2}-\frac{2}{3} K+\frac{1}{3} \sqrt{3-6 K+4 K^{2}-12 u K_{o}} \\
r_{2}^{s *}=\frac{1}{2}-\frac{2}{3} K-\frac{1}{3} \sqrt{3-6 K+4 K^{2}-12 u K_{o}}
\end{array} .\right.
$$

Ces deux solutions sont telles que $r_{2}^{s *}<r_{1}^{s *} ; r_{2}^{s *}$ se situe dans la partie croissante et $r_{1}^{s *}$ dans la partie décroissante du profit. Ces deux racines existent si $3-6 K+4 K^{2}-12 u K_{o} \geq 0 \Leftrightarrow u K_{o} \leq \frac{1}{4}-\frac{1}{2} K+\frac{1}{3} K^{2}$. Cette inégalité est possible puisque $\frac{1}{4}-\frac{1}{2} K+\frac{1}{3} K^{2}$ est toujours strictement positif (racines complexes).

Ce terme $\frac{1}{4}-\frac{1}{2} K+\frac{1}{3} K^{2}$ est de plus dans notre intervalle de variation de $u K_{o}$, c'est-à-dire $\left[0, \frac{1}{4}\right]$. Il peut donc exister un intervalle $u K_{o} \in$ $\left[\frac{1}{4}-\frac{1}{2} K+\frac{1}{3} K^{2}, \frac{1}{4}\right]$ dans lequel le régulateur ne peut adapter sa politique 
de régulation $\left(\alpha^{s}, r^{s}\right)$ pour éviter et financer les coûts échoués irrécupérables, les prix $r_{1}^{s *}$ et $r_{2}^{s *}$ n'existant pas.

Le régulateur, pour maximiser le welfare, choisira toujours le plus petit prix possible atteignable. Les deux racines existent si $u K_{o} \leq \frac{1}{4}-\frac{1}{2} K+\frac{1}{3} K^{2}$. Par conséquent, $r_{2}^{s *}<r_{1}^{s *}$, le régulateur ne choisira jamais $r_{1}^{s *}$, qui de plus est supérieur à $\frac{1}{6}$.

$r_{2}^{s *}$ est toujours supérieur à $\frac{1}{6}: r_{2}^{s *}-\frac{1}{6}>0 \Leftrightarrow u K_{o}>\frac{1}{6}-\frac{1}{6} K$. Par conséquent, pour $u K_{o}>\frac{1}{6}-\frac{1}{6} K$, il choisira toujours le prix de rétrocession $r_{2}^{s *}$ qui est le plus petit prix possible tel que $\Pi_{o}^{s}$ est non négatif.

La variable duale associée à ce prix $r_{2}^{s *}$ sera égale à $\gamma_{2}^{s *}=\frac{2 r_{2}^{s *}+1}{6-8 K-12 r_{2}^{s *}}$. Pour que $r_{2}^{s *}$ soit solution, il faut que ce multiplicateur soit positif. Ce multiplicateur est positif pour un prix de rétrocession inférieur à $r^{s \max }=\frac{1}{2}-\frac{2}{3} K$, ce qui est toujours vérifié pour $r_{2}^{s *}$.

Les conditions d'existence de $r_{2}^{s}$ sont $u K_{o} \leq \frac{1}{4}-\frac{1}{2} K+\frac{1}{3} K^{2}$. Il est nécessaire de vérifier sous quelles conditions $\frac{1}{6}-\frac{1}{6} K<u K_{o}$ se vérifient aussi. La différence entre les deux termes précédents nous conduit à calculer une racine double $K=\frac{1}{2}$. Cette différence est donc toujours positive pour $K \leq \frac{1}{2}$ ce qui nous donne $\frac{1}{4}-\frac{1}{2} K+\frac{1}{3} K^{2}>\frac{1}{6}-\frac{1}{6} K$.

Les deux prix d'équilibre $r^{s *}=\frac{1}{6}$ et $r^{s *}=r_{2}^{s *}$ vérifient bien la condition d'équilibre du jeu complet $K \leq \frac{1}{2}-r^{s}$ :

- Si $r^{s *}=\frac{1}{6}$, alors cette condition nous donne $K \leq \frac{1}{3}$ ce qui est intuitif puisque le concurrent ne peut pas jouer sa stratégie de meilleure réponse non contrainte, c'est-à-dire $q_{e}^{*}=\frac{1}{3}$ en ne s'approvisionnant qu'auprès de la source alternative.

- Si $r^{s *}=r_{2}^{s *}$ alors $r^{s} \leq \frac{1}{2}-K \Leftrightarrow r_{2}^{s *} \leq \frac{1}{2}-K \Leftrightarrow \frac{1}{4}(K-1)^{2} \geq u K_{o}$. Positionnons ce nouveau seuils par rapport à $\frac{1}{4}-\frac{1}{2} K+\frac{1}{3} K^{2}$ (seuil pour lequel $r_{2}^{s *}$ existe) et $\frac{1}{6}-\frac{1}{6} K$ (seuil pour lequel les profit de l'OH sont négatifs avec un prix de rétrocession $r^{s *}=\frac{1}{6}$ ). Il est toujours inférieur à $\frac{1}{4}-\frac{1}{2} K+\frac{1}{3} K^{2}$ et pour $K \leq \frac{1}{3}, \frac{1}{4}(K-1)^{2} \geq \frac{1}{6}-\frac{1}{6} K$.

Finalement, plusieurs cas sont à envisager pour déterminer l'existence des solutions : 
- Si $\frac{1}{6}-\frac{1}{6} K \geq u K_{o}$, alors $r_{2}^{s *} \leq \frac{1}{6}$ le régulateur choisit $r^{s *}=\frac{1}{6}$ et permet à l'OH de réaliser des profits positifs en raison de l'efficacité de ses conditions d'approvisionnement ( $u$ efficace).

- Si $\frac{1}{4}(K-1)^{2} \geq u K_{o}>\frac{1}{6}-\frac{1}{6} K$, alors $r_{2}^{s *} \geq \frac{1}{6}$ et le régulateur choisit $r_{2}^{s *}$ qui permet à l'OH de réaliser un profit nul.

- Si $u K_{o}>\frac{1}{4}(K-1)^{2}$, alors la contrainte d'existence de l'équilibre $K \leq$ $\frac{1}{2}-r^{s}$ n'est pas vérifiée.

Le welfare est croissant en quantités jouées sur le marché aval. Par conséquent, le régulateur choisit une proportion minimale $\alpha^{s}$ telle que le concurrent ne soit pas contraint : $\left(\alpha^{s *} K_{o}+K\right)=\frac{1}{2}-r^{s *}$ soit vérifiée, c'est-à-dire $\left(\alpha^{s *} K_{o}+K\right)=\frac{1}{2}-r^{s *} \Leftrightarrow \alpha^{s *}=\frac{1-2 r^{s *}-2 K}{2 K_{o}}$. Cette proportion est comprise dans l'intervalle $[0,1]$ car elle est :

- positive pour $K_{e}^{s *}>0: \alpha^{s *}>0 \Leftrightarrow \frac{1}{2}-K-r^{s}>0 \Leftrightarrow K_{e}^{s *}>0$;

- inférieure à $1: \alpha^{s *}<1 \Leftrightarrow \frac{1}{2}-K-K_{o}<r^{s}$. Or on sait que $\left(\alpha^{s} K_{o}+K\right) \geq$ $\frac{1}{2}-r^{s}$ pour cet équilibre donc $\frac{1}{2}-K-K_{o}<r^{s}$ est toujours le cas puisque $\alpha^{s} K_{o}<K_{o}$.

Si le régulateur choisit $r^{s *}=\frac{1}{6}$, alors $\alpha^{s}=\frac{1-3 K}{3 K_{o}}$ positive pour $K<\frac{1}{3}$ ce qui est le cas pour être à cet équilibre. Donc il peut choisir une proportion minimale $\alpha^{s *}=\frac{1-3 K}{3 K_{o}}$. Si le régulateur choisit $r^{s *}=r_{2}^{s *}$, alors il choisira une proportion $\alpha^{s *}=\alpha_{2}^{s *}=\frac{1}{3} \frac{\sqrt{3-6 K+4 K^{2}-12 u K_{o}}-K}{K_{o}}$.

Pour que le mécanisme de rétrocession soit efficace, le concurrent doit réaliser des profits positifs pour les deux prix de rétrocession $\Leftrightarrow \Pi_{e}^{s *}=$ $p^{s *}\left(q_{o}^{s *}\right) q_{e}^{s *}-r^{s *} K_{e}^{s *}-s K_{s}^{*}>0$. L'expression développée nous donne $\Pi_{e}^{s *}=$ $K r^{s *}-\frac{1}{2} r^{s *}-K s+\frac{1}{2} r^{s * 2}+\frac{1}{8}$. La dérivée du profit du concurrent $\Pi_{e}^{s *}$ est décroissante par rapport au prix de rétrocession : $\frac{d \Pi_{e}^{s}}{d r^{s}}=K+r^{s}-\frac{1}{2} \leq 0$ à l'équilibre car $K_{e}^{s *}=\frac{1}{2}-K-r^{s} \geq 0$. Les limites aux bornes de l'intervalle de variation du prix de rétrocession nous indiquent que ce profit est toujours positif pour un prix de rétrocession $r^{s *} \in\left[\frac{1}{6}, \frac{1}{2}\right]: \operatorname{Lim}_{r^{s *} \rightarrow \frac{1}{2}} \prod_{e}^{s *}=\frac{1}{2} K-K s$ positif pour $s<\frac{1}{2}$.

\section{La contrainte $K_{e}^{s}=\alpha^{s} K_{o}$ est seule saturée}


L'équilibre en sous-jeu est $\left(q_{e 3}^{s *}, q_{o 3}^{s *}\right)$. La fonction de bien-être est ici indépendante du prix de rétrocession, la contrainte de régulation asymétrique étant saturée : $W_{3}^{s}=\frac{15}{32}-\frac{5}{8} s+\frac{7}{8} s^{2}-u K_{o}+s \alpha^{s} K_{o}$.

C'est une fonction croissante en quantités rétrocédée : $\frac{d W_{3}^{s}}{d \alpha^{s}}=s K_{o}>0$ donc le welfare est croissant en $\alpha^{s}$.

La condition de positivité sur les approvisionnements $K_{s}^{*}$ nous permet de déterminer une condition sur la proportion rétrocédée $\alpha^{s}: K_{s} \geq 0 \Leftrightarrow$ $\frac{1-2 s}{2 K_{o}} \geq \alpha$. Le welfare étant croissant en $\alpha$, le régulateur sera incité à fixer la valeur maximale, c'est-à-dire $\alpha^{s *}=\frac{1-2 s}{2 K_{o}}$. Fixer cette proportion est possible car $\alpha^{s *} K_{o}<K_{o} \Leftrightarrow \frac{1-2 s}{2}<K_{o} \Leftrightarrow q_{e}^{s *}<K_{o}$ ce qui est toujours vérifié. En fixant cette proportion, le régulateur permet au concurrent de s'approvisionner à la source la moins chère, c'est-à-dire auprès de la $\mathrm{RA}: K_{s}^{*}=0$ et $K_{e}^{s *}=\alpha^{s *} K_{o}$.Sans tenir compte des profits de l'OH, le régulateur peut fixer un prix de rétrocession respectant $r^{s} \leq s$. Dans ce cas, fixer un prix de rétrocession au coût marginal de l'OH est possible. Cependant, comme dans les équilibres précédents, l'OH est susceptible d'encourir des coûts échoués irrécupérables que le régulateur est incité à financer pour éviter la monopolisation de l'activité. Le prix de rétrocession peut donc être une solution Ramsey-Boîteux, vérifiant $\Pi_{o}^{s}=0 \Leftrightarrow r^{s}+\frac{1}{4} s-u K_{o}-2 r^{s} s+\frac{1}{4} s^{2}+\frac{1}{16}=0$.

Les profits de l'OH sont croissants en $r^{s}: \frac{d \Pi_{o}^{s}}{d r^{s}}=1-2 s>0$ pour $s<\frac{1}{2}$ ce qui est toujours vérifié.

Le profit est ici une fonction du premier degré du prix de rétrocession. Le seul prix de rétrocession qui permet d'atteindre un profit nul sera : $\Pi_{o}^{s}=$ $0 \Leftrightarrow r_{3}^{s *}=\frac{-4 s+16 u K_{o}-4 s^{2}-1}{16-32 s}$. Ce prix est du signe de $-4 s+16 u K_{o}-4 s^{2}-1$ car $s<\frac{1}{2}$. Ce terme dispose de deux racines : $-4 s+16 u K_{o}-4 s^{2}-1=0 \Leftrightarrow$ $\left\{\begin{array}{c}s_{1}=2 \sqrt{u K_{o}}-\frac{1}{2} \\ s_{2}=-2 \sqrt{u K_{o}}-\frac{1}{2}\end{array}\right.$.

La racine $s_{2}$ est toujours négative. La racine $s_{1}$ est positive si $2 \sqrt{u K_{o}}-\frac{1}{2}>$ $0 \Leftrightarrow u K_{o}>\frac{1}{16}$. Elle est toujours inférieure à $\frac{1}{2}\left(u K_{o} \leq \frac{1}{4}\right)$ et supérieure à $\frac{1}{6}$ si $u K_{o} \geq \frac{1}{9}$.

Viennent alors plusieurs cas de discussion : 
- Si $u K_{o} \geq \frac{1}{9}$, alors $s_{1} \geq \frac{1}{6}$. Si $s \in\left[\frac{1}{6}, s_{1}\right]$ alors $r_{3}^{s *}>0$. Si $s \in\left[s_{1}, \frac{1}{2}\right]$ alors $r_{3}^{s *}<0$.

- Si $u K_{o}<\frac{1}{9}$, alors $s_{1}<\frac{1}{6} \operatorname{donc} r_{3}^{s *}<0$.

Le profit de l'OH est croissant en $r^{s}$ ce qui nous conduit à des profits positifs sauf lorsque $s \in\left[\frac{1}{6}, s_{1}\right]$.

Le prix $r_{3}^{s *}$ dépend du prix $s$. Vérifions désormais que $r_{3}^{s *}$ est inférieur à $s$ pour que l'équilibre soit possible. La différence $r_{3}^{s *}-s=\frac{28 s^{2}-20 s+16 u K_{o}-1}{16-32 s}$ est du signe de $28 s^{2}-20 s+16 u K_{o}-1$. Ce terme dispose de deux racines $\left\{\begin{array}{l}s_{3}=\frac{2}{7} \sqrt{2-7 u K_{o}}+\frac{5}{14} \\ s_{4}=\frac{5}{14}-\frac{2}{7} \sqrt{2-7 u K_{o}}\end{array}\right.$.

La racine $s_{3}$ est toujours positive, $s_{3}>0 \Leftrightarrow \frac{2}{7} \sqrt{2-7 u K_{o}}>-\frac{5}{14}$, mais toujours supérieure à $\frac{1}{2}$, borne supérieure de notre intervalle de variation de $s\left(s_{3}-\frac{1}{2}>0\right.$ car $\left.u K_{o} \leq \frac{1}{4}\right)$.

La racine $s_{4}=\frac{5}{14}-\frac{2}{7} \sqrt{2-7 u K_{o}}$ est positive pour $\frac{1}{16}<u K_{o}$ et toujours inférieure à $\frac{1}{2}: s_{4}-\frac{1}{2}<0 \Leftrightarrow-\frac{2}{7} \sqrt{2-7 u K_{o}}-\frac{1}{7}<0$.

Vérifions maintenant sa position par rapport à la borne inférieure de l'intervalle $\frac{1}{6}: s_{4}-\frac{1}{6}=\frac{5}{14}-\frac{2}{7} \sqrt{2-7 u K_{o}}-\frac{1}{6}>0$ si $u K_{o}>\frac{2}{9}$.

Finalement, plusieurs cas sont à considérer pour la discussion :

- Si $u K_{o}<\frac{1}{9}$, alors $r_{3}^{s *}<0$ donc le régulateur choisit un prix $r^{s *}=0$ ou $r^{s *}=u$. Le profit de l'OH est positif et tout prix $r^{s *}<s$ n'agit que sur la répartition des profits entre $\mathrm{OH}$ et concurrent.

- Si $\frac{2}{9} \geq u K_{o} \geq \frac{1}{9}$, alors deux situations existent :

- si $s \in\left[\frac{1}{6}, s_{1}\right]$, alors $s>r_{3}^{s *} \geq 0$ donc le régulateur le choisit comme prix de rétrocession d'équilibre $: r^{s *}=r_{3}^{s *}$.

- si $\left.s \in] s_{1}, \frac{1}{6}\right]$, alors $r_{3}^{s *}<0$ donc le régulateur choisit un prix $r^{s *}=0$ ou $r^{s *}=u$. Le profit de l'OH est positif et tout prix $r^{s *}<s$ n'agit que sur les répartitions de profits entre $\mathrm{OH}$ et concurrent.

- Si $\frac{1}{4} \geq u K_{o}>\frac{2}{9}$, alors trois cas existent :

- si $s \in\left[\frac{1}{6}, s_{4}\left[, r_{3}^{s *}>s\right.\right.$ donc le régulateur ne peut pas choisir le prix qui permet de financer les coûts échoués irrécupérables. L'OH est trop inefficace pour qu'il puisse réaliser des profits positifs, sachant que 
les prix sur le marché alternatif ne sont pas très élevés. Il établit un prix $r^{s}<s$ mais l'OH réalise des pertes.

- si $s \in\left[s_{4}, s_{1}\right], r_{3}^{s *} \leq s$ donc le régulateur peut financer les coûts échoués irrécupérables de l'OH sachant que les prix de la source alternative sont plus élevés. Le prix d'équilibre est donc $r^{s *}=r_{3}^{s *}$.

- si $\left.s \in] s_{1}, \frac{1}{2}\right], r_{3}^{s *}<0$ donc le régulateur choisit un prix $r^{s *}=0$ ou $r^{s *}=u$. Le profit de l'OH est positif car les prix de la source alternative sont très élevés. Tout prix $r^{s *}<s$ n'agit que sur les répartitions de profits entre $\mathrm{OH}$ et concurrent.

Regardons si pour ce prix $r^{s *}=r_{3}^{s *}$ le profit du concurrent est positif. Le profit du concurrent est positif pour $r \leq \frac{1}{4}+\frac{1}{2} s: \Pi_{e}=\left(\frac{1}{4}+\frac{1}{2} s-r\right)\left(\frac{1}{2}-s\right)$ $\geq 0$ pour $r \leq \frac{1}{4}+\frac{1}{2} s$.

Il faut donc que $r_{3}^{s *}$ soit inférieur au prix sur le marché final pour que la RA soit efficace : $r_{3}^{s *}-\left(\frac{1}{4}+\frac{1}{2} s\right)=\frac{1}{16-32 s}\left(12 s^{2}-4 s+16 u K_{o}-5\right)$ expression qui doit être négative pour que $r_{3}^{s *}$ soit solution.

Cette expression est du signe de $12 s^{2}-4 s+16 u K_{o}-5$. Deux racines l'annulent : $\left\{\begin{array}{l}s_{a}=\frac{2}{3} \sqrt{1-3 u K_{o}}+\frac{1}{6} \\ s_{b}=\frac{1}{6}-\frac{2}{3} \sqrt{1-3 u K_{o}}\end{array}\right.$. Vérifions où se situent ces deux racines dans l'intervalle d'équilibre de $s \in\left[\frac{1}{6}, \frac{1}{2}\right] . s_{b} \geq 0 \Leftrightarrow u K_{o} \geq \frac{5}{16}$ impossible car par hypothèse $u K_{o} \leq \frac{1}{4}$ donc $s_{b}<0 . s_{a} \geq \frac{1}{2} \Leftrightarrow u K_{o} \leq \frac{1}{4}$ ce qui est toujours vérifié. Par conséquent, $12 s^{2}-4 s+16 u K_{o}-5$ est négative puisque $s \in\left[\frac{1}{6}, \frac{1}{2}\right]$ se situe entre les deux racines. Le concurrent, pour un prix $r^{s *}=r_{3}^{s *}$, réalise toujours un profit qui est positif. 